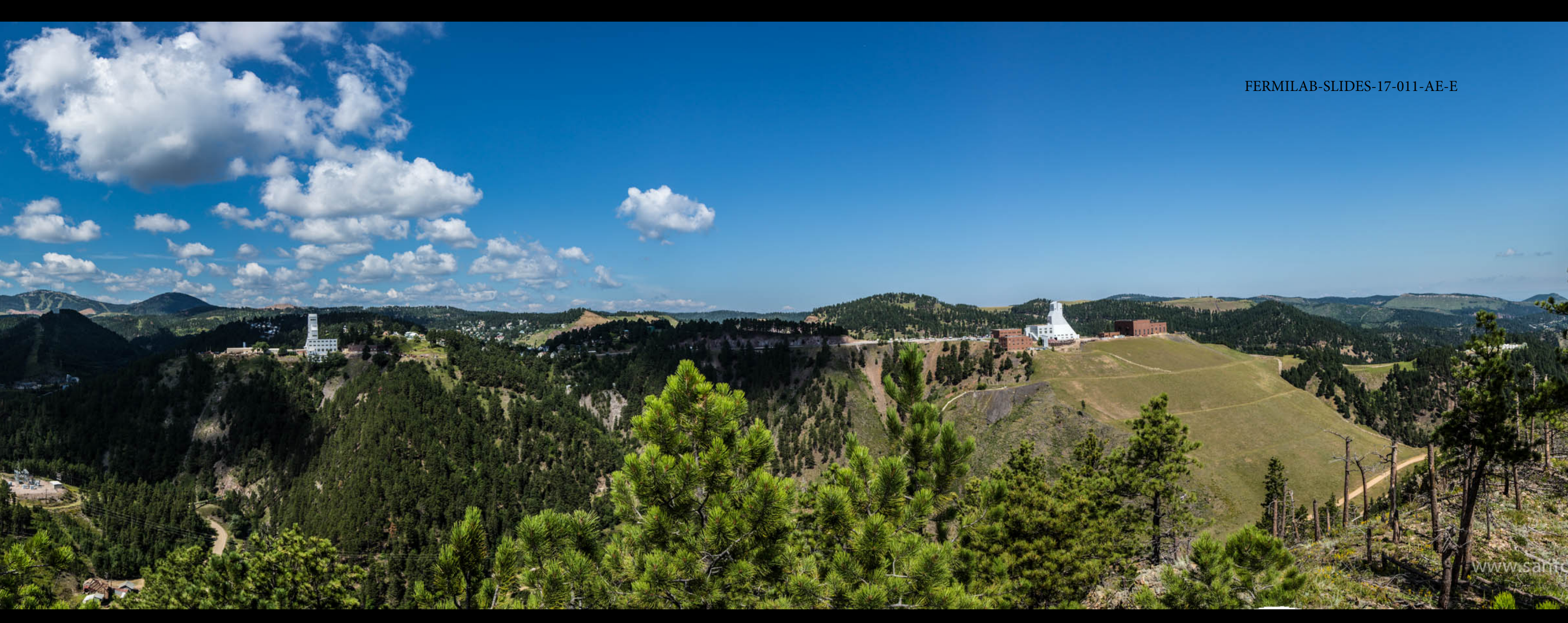

\title{
Searching for Dark Matter with LZ
}

Hugh Lippincott, Fermilab for the LZ Collaboration

\author{
Exploring the Dark Universe \\ July, 2017
}




\section{LXe as Dark Matter Target}

\begin{tabular}{|c|c|c|}
\hline Challenge & Solution & Liquid Xenon \\
\hline Extremely rare & Large mass & $\begin{array}{c}\text { Very dense }-3 \text { tonnes } \\
\text { in } 1 \mathrm{~m}^{3}\end{array}$ \\
\hline $\begin{array}{c}\text { Energy depositions of } \\
\sim 10 \text { keV or below }\end{array}$ & $\begin{array}{c}\text { Low energy } \\
\text { thresholds }\end{array}$ & $\begin{array}{c}\sim 60-70 \text { electrons }+ \\
\text { photons } / \text { keV }\end{array}$ \\
\hline $\begin{array}{c}\text { Backgrounds - } \\
\text { Impurities }\end{array}$ & Purification & $\begin{array}{c}\text { Noble gases are } \\
\text { (mostly) easy to purify }\end{array}$ \\
\hline $\begin{array}{c}\text { Backgrounds - } \\
\text { Detector }\end{array}$ & Self shielding & $\begin{array}{c}\text { Low MFP for ionizing } \\
\text { radiation }\end{array}$ \\
\hline $\begin{array}{c}\text { Backgrounds - } \\
\text { Internal/Detector }\end{array}$ & Discrimination & $\begin{array}{c}\text { Charge to light ratio } \\
\text { gives particle ID }\end{array}$ \\
\hline
\end{tabular}




\section{Two phase Xenon Detectors}

- Interaction in the xenon creates:

- Scintillation light ( 10 ns)

- called SI

- ionization electrons

- Electrons drift through electric field to liquid/gas surface

- Extracted into gas and accelerated creating proportional scintillation light - called S2

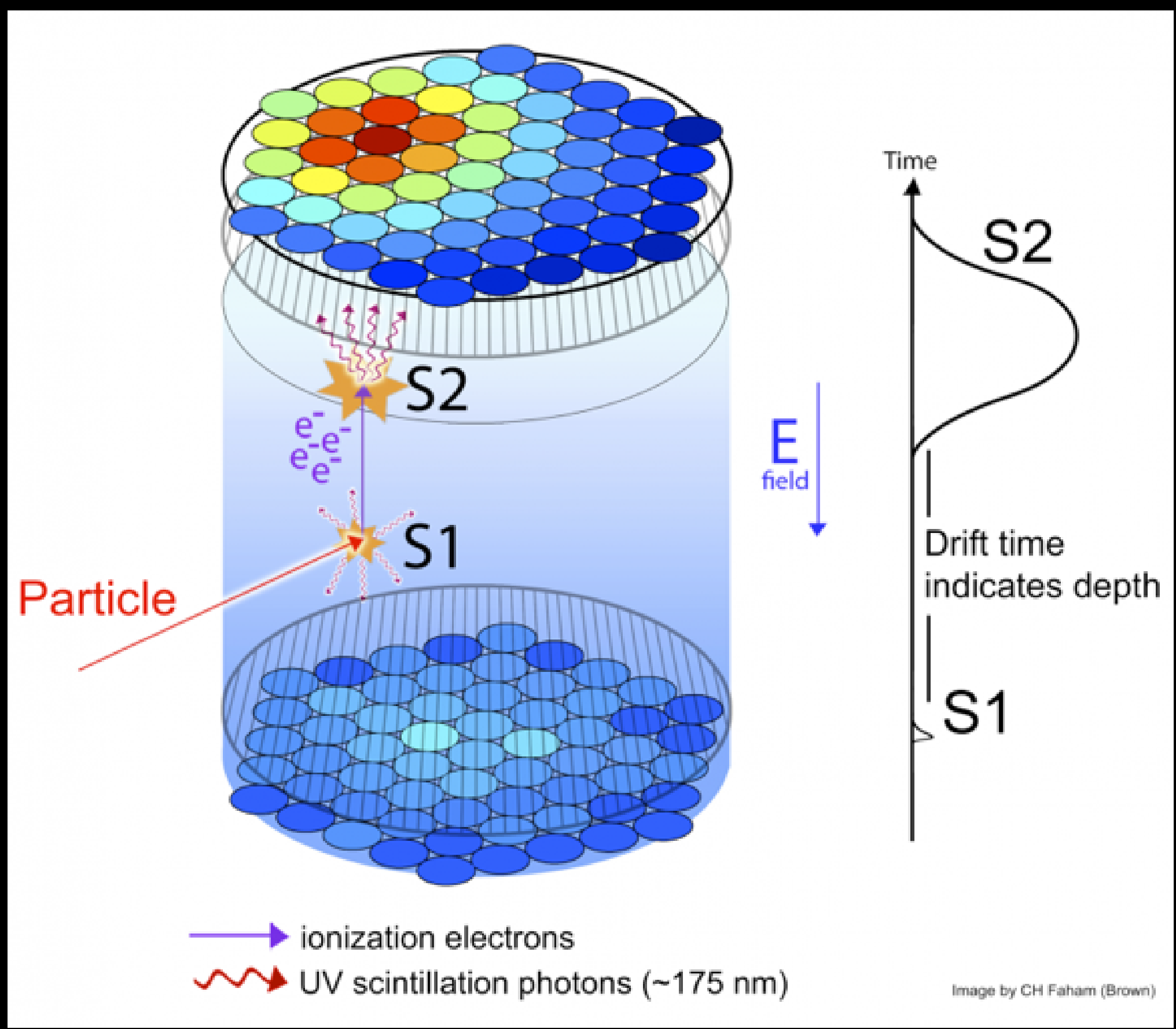




\section{Two phase Xenon Detectors}

- Excellent 3D reconstruction ( $\sim \mathrm{mm})$

- Z position from SI-S2 timing

- XY position from hit pattern of S2 light

- Allows for self shielding, rejection of edge events

- Ratio of charge (S2) to light (SI) gives particle ID

- Better than $99.5 \%$

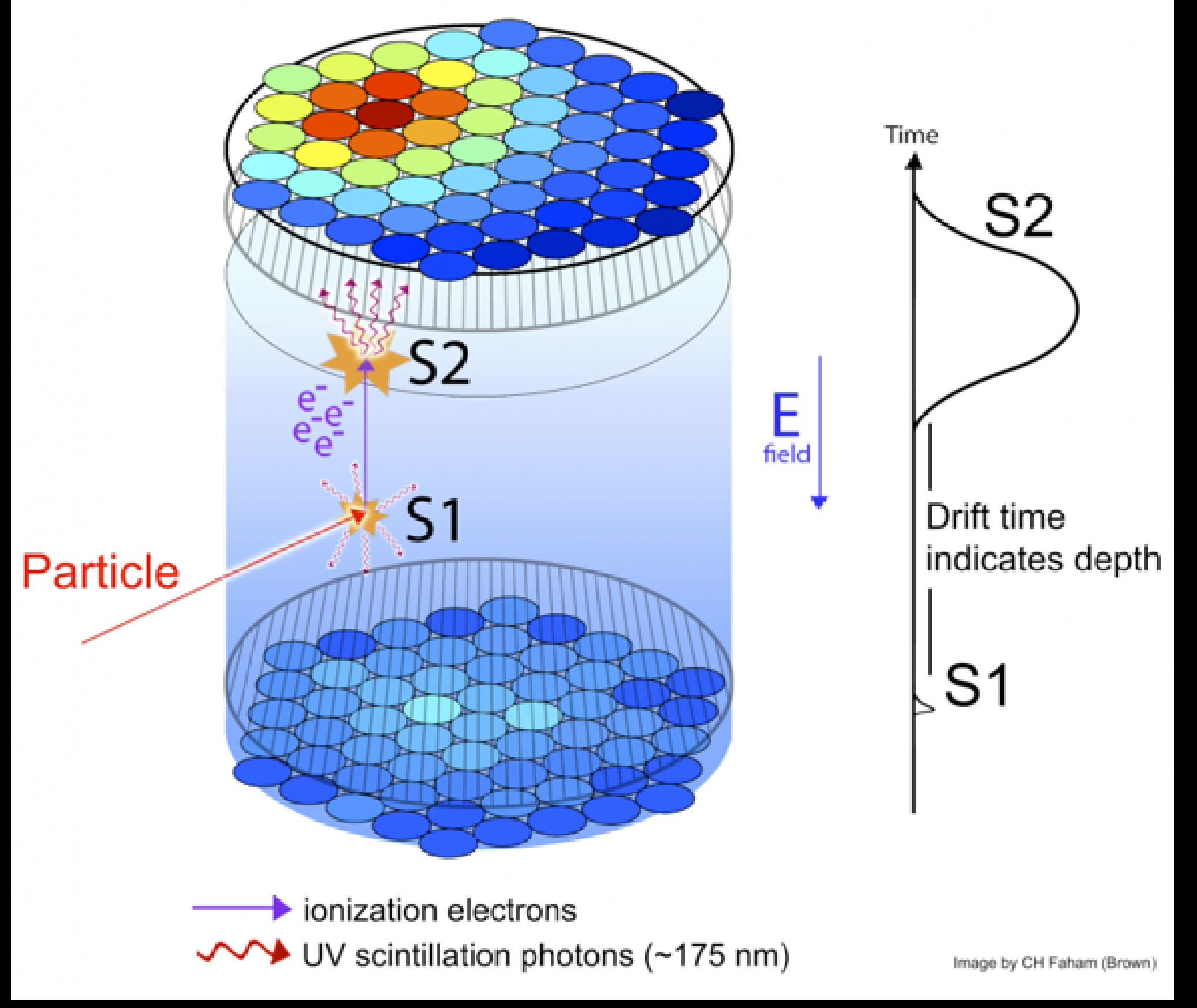
rejection of electron recoil (ER) events 


\section{Self shielding is powerful}

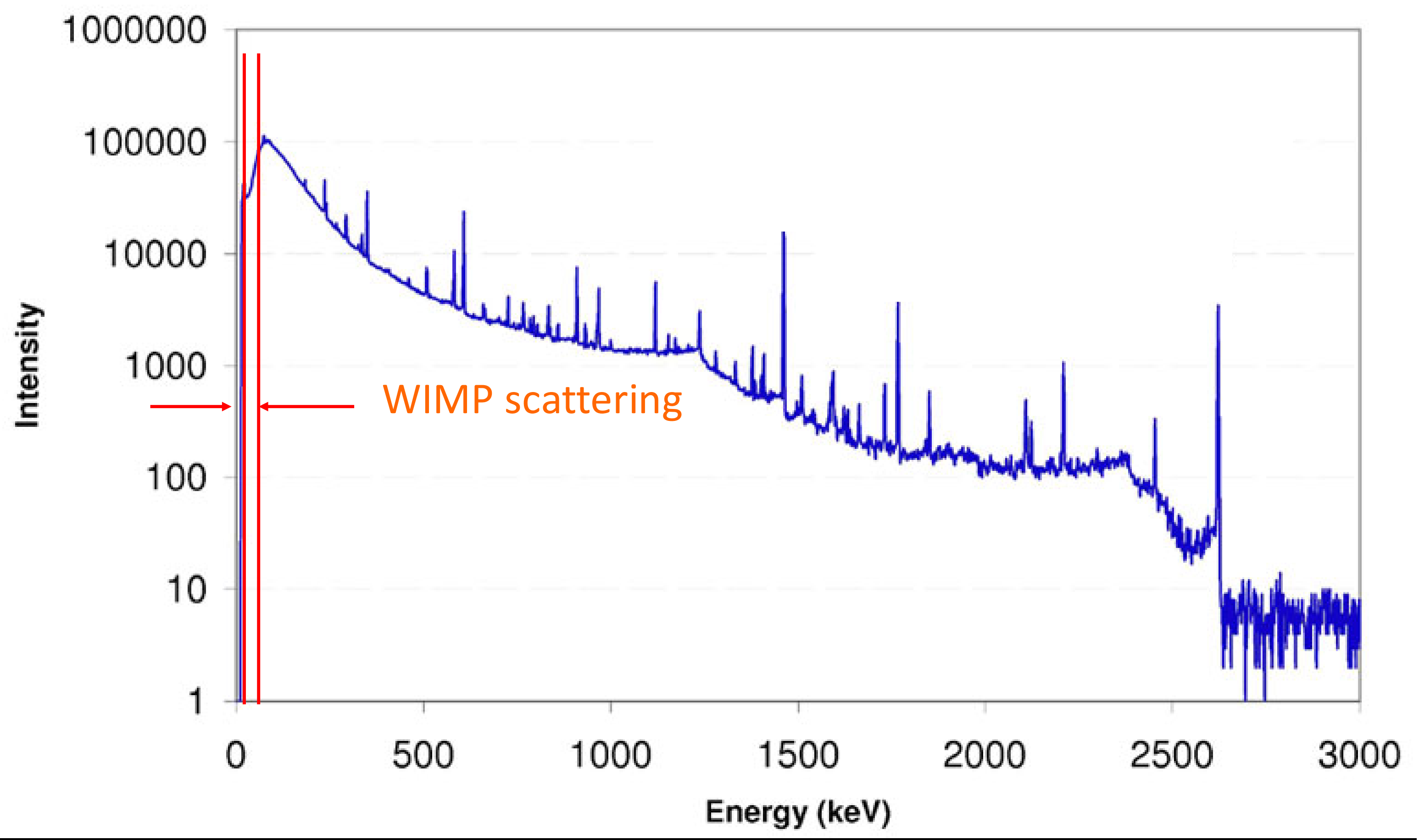




\section{Self shielding is powerful}

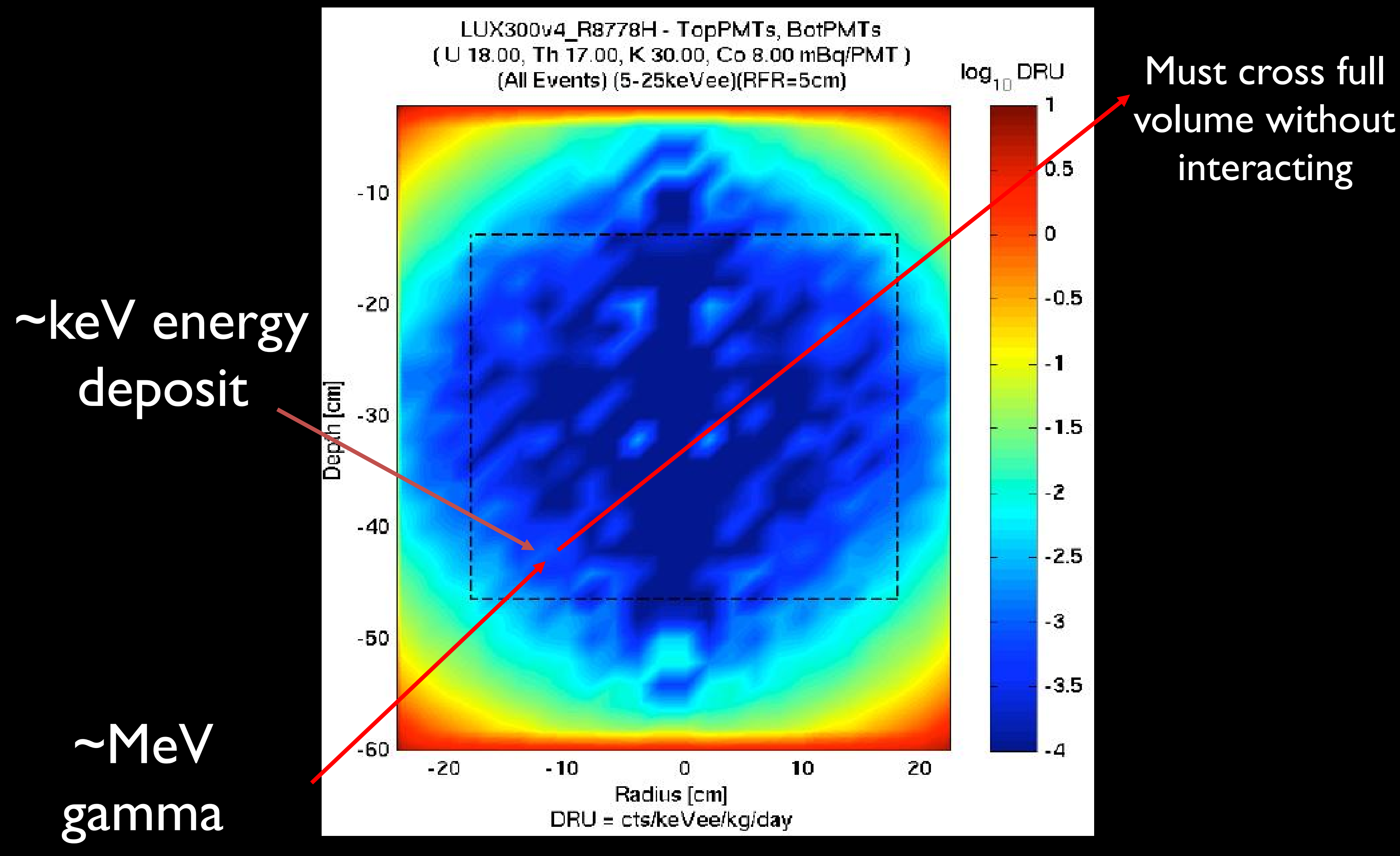




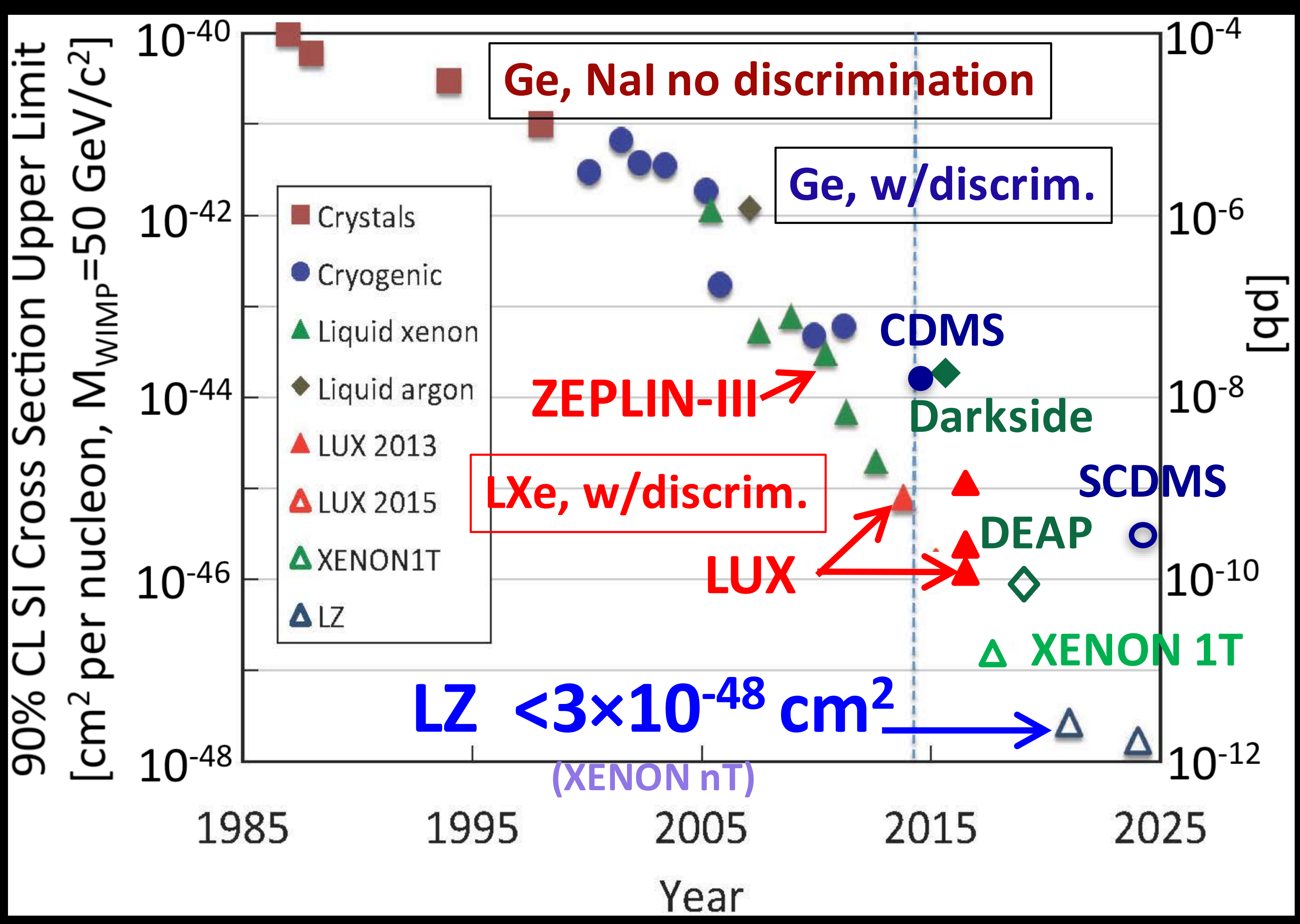






arXiv:|705.06655 WIMP mass $\left[\mathrm{GeV} / \mathrm{c}^{2}\right]$

$$
\begin{aligned}
& \text { LUX - } 100 \text { kg (active) } \\
& \text { PandaX-II - } 329 \text { kg (fid) } \\
& \text { Xenon IT - I tonne (fid) }
\end{aligned}
$$




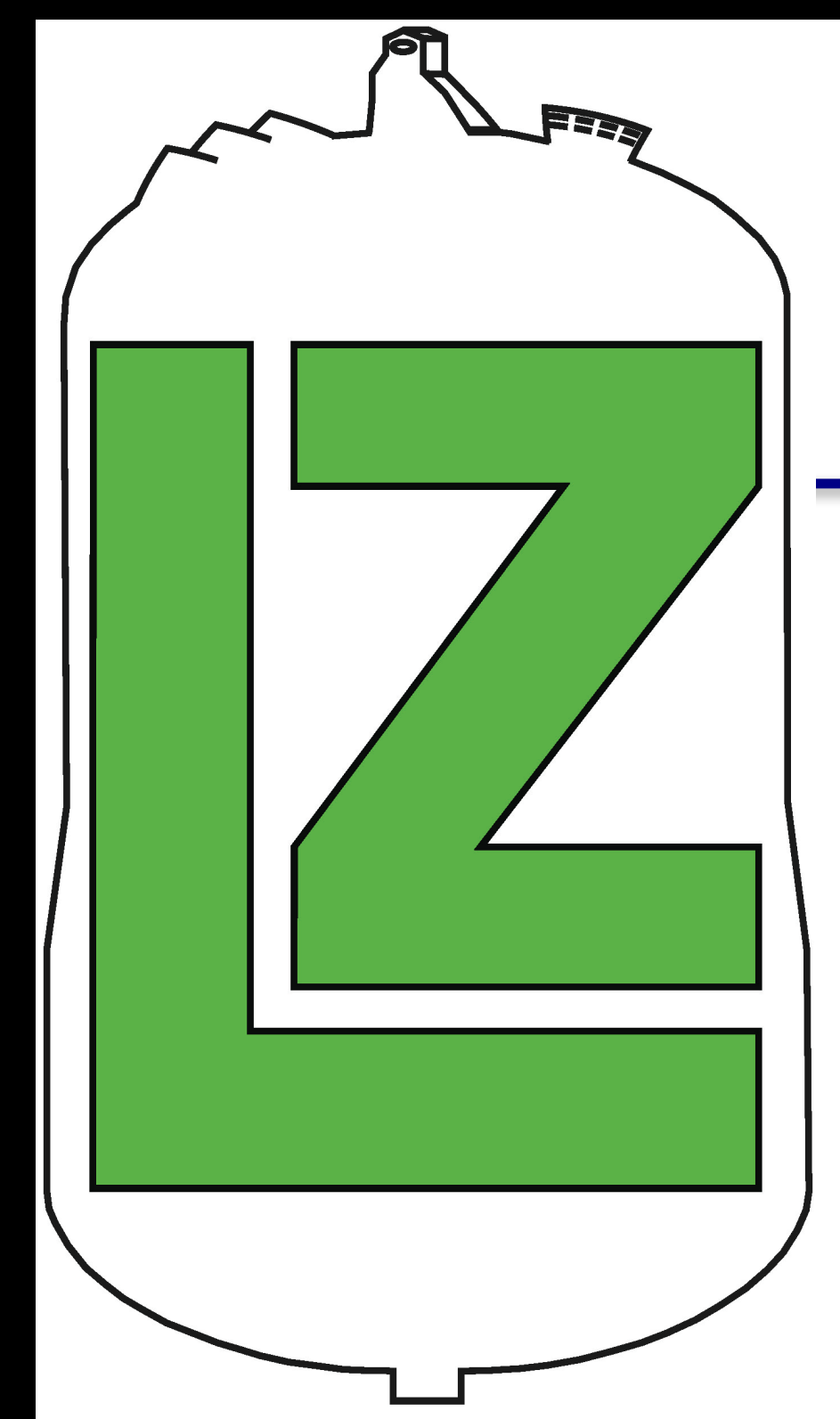

Center for Underground Physics (Korea) Imperial College London (UK) LIP Coimbra (Portugal) MEPhI (Russia)

STFC Rutherford Appleton Laboratory (UK) University College London (UK) University of Bristol (UK) SUPA, University of Edinburgh (UK) University of Liverpool (UK) University of Oxford (UK) University of Sheffield (UK)

\section{$\mathrm{LZ}=\mathrm{LUX}+\mathrm{ZEPLIN}$}

\section{Institutions, 217 People}

Black Hills State University

Brookhaven National Laboratory (BNL)

Brown University

Fermi National Accelerator Laboratory (FNAL)

Kavli Institute for Particle Astrophysics and Cosmology (KIPAC)

Lawrence Berkeley National Laboratory (LBNL)

Lawrence Livermore National Laboratory (LLNL)

Northwestern University

Pennsylvania State University

SLAC National Accelerator Laboratory

South Dakota School of Mines and Technology

South Dakota Science and Technology Authority (SDSTA)

STFC Rutherford Appleton Laboratory (RAL)

Texas A\&M University

University at Albany (SUNY)

University of Alabama

University of California (UC), Berkeley

University of California (UC), Davis

University of California (UC), Santa Barbara

University of Maryland

University of Massachusetts
University of Michigan University of Rochester University of South Dakota University of Wisconsin-Madison Washington University in St. Louis Yale University 


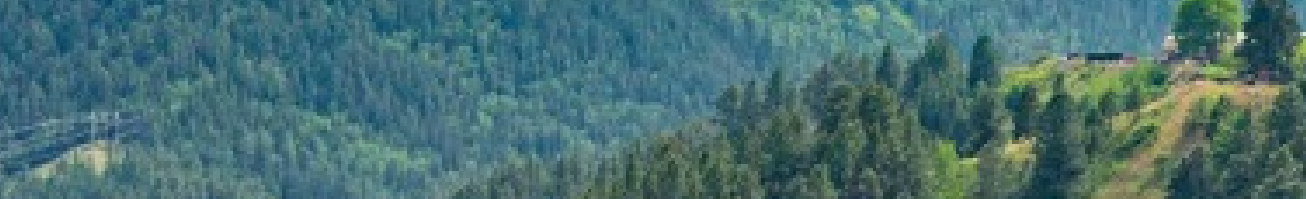

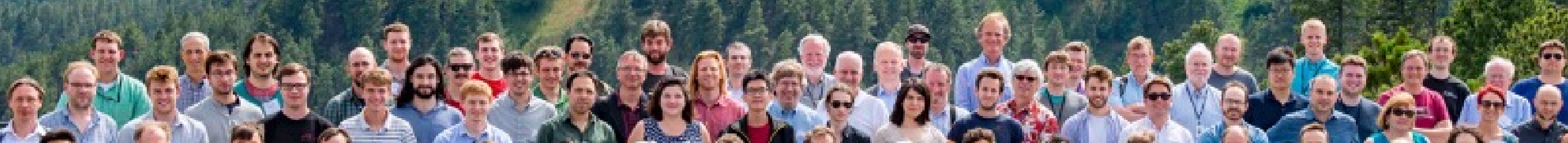

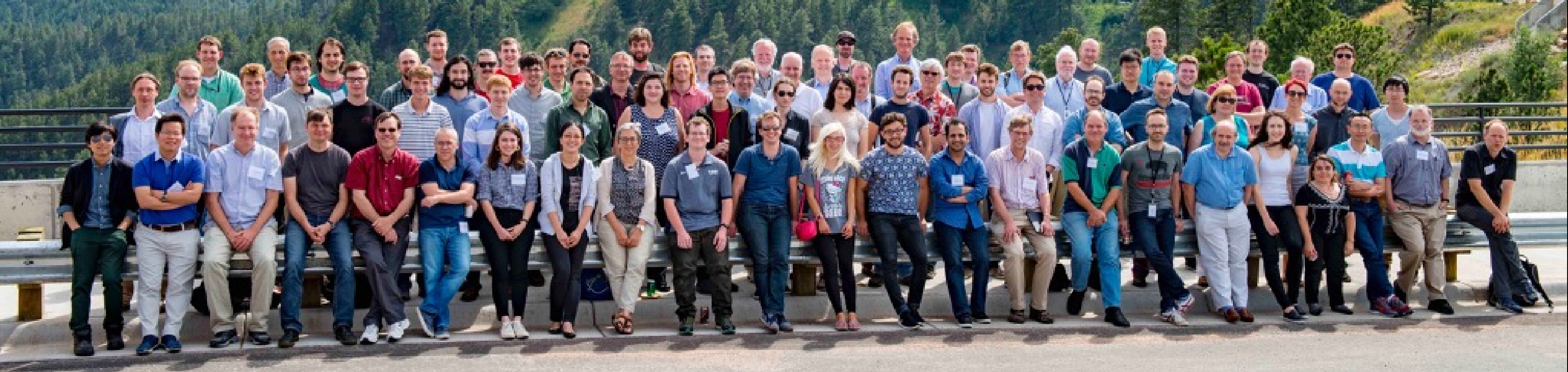

Collaboration meeting last week at SURF 


\section{Scale Up $\approx 50$ in Fiducial Mass}

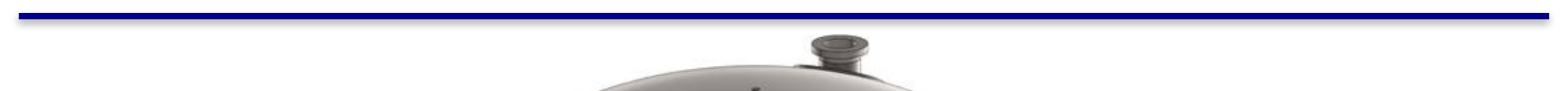

Total mass $-10 \mathrm{~T}$ WIMP Active Mass - $7 \mathrm{~T}$

WIMP Fiducial Mass - 5.6 T 


\section{Sanford Underground Research Facility}

\section{Davis Cavern 1480 m (4200 mwe) LUX Water Tank}




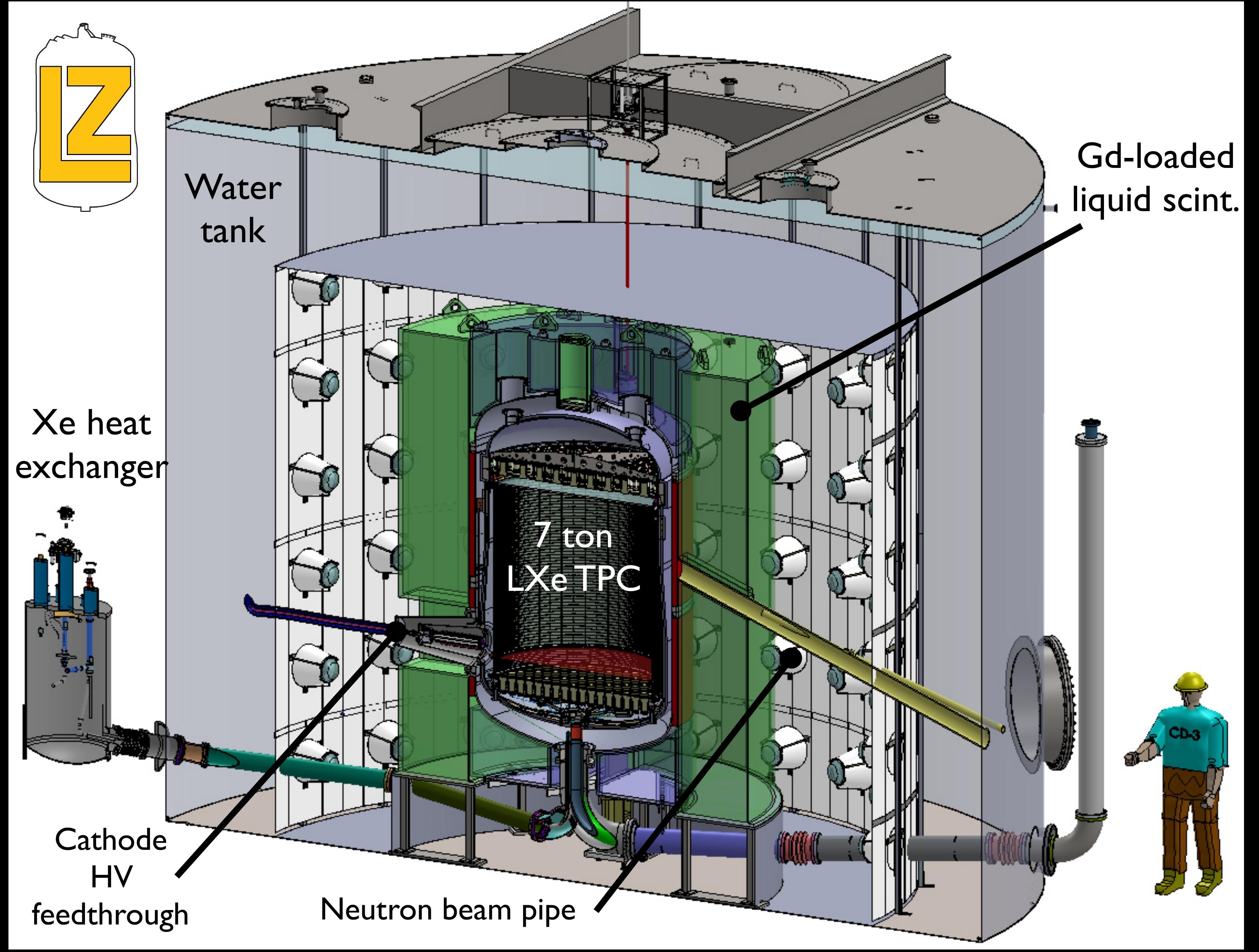




\section{LZ design notes}

- More mass (x50 more than LUX, x6 more than Xenon IT)

- 494 3" PMTs on TPC

- Significant HV/grid engineering (no xenon experiment has achieved HV goals so far)

- Requirement: 50 kV Goal: 100 kV

- Sophisticated veto system - maximizes fiducial volume

- LXe “skin" - 93 I" PMTs + 38 2" PMTs

- 120 outer detector PMTs

- Radioactivity, radioactivity, radioactivity! 


\section{System test at SLAC}

- Main test platform for LZ

- Same cryogenics/control

- Phase I (ongoing)

- Full LZ fields in scaled prototype TPC

- Can HV be achieved with sparking or light emission?

- Prototype circulation

- LZ architecture and compressor

- Phase II will test grids

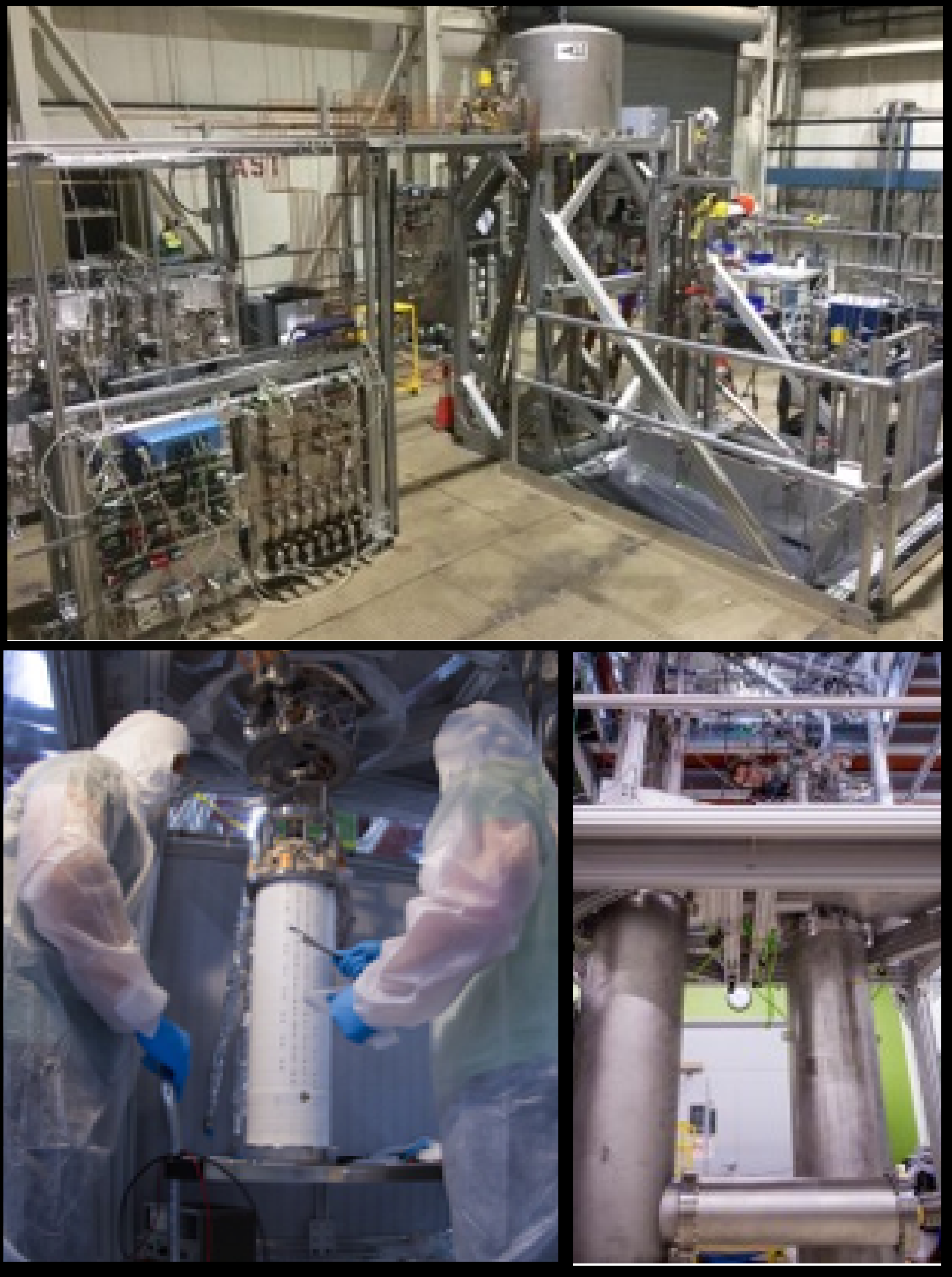




\section{Background suppression by screening}

- Every component is screened and simulated for radioactivity

- E.g. cryostat made of the most radiopure titanium in the world: $<0.05$ counts in 1000 days after cuts

- Similar campaign working with Hamamatsu on PMTs

- Backed up by extensive quality assurance during production

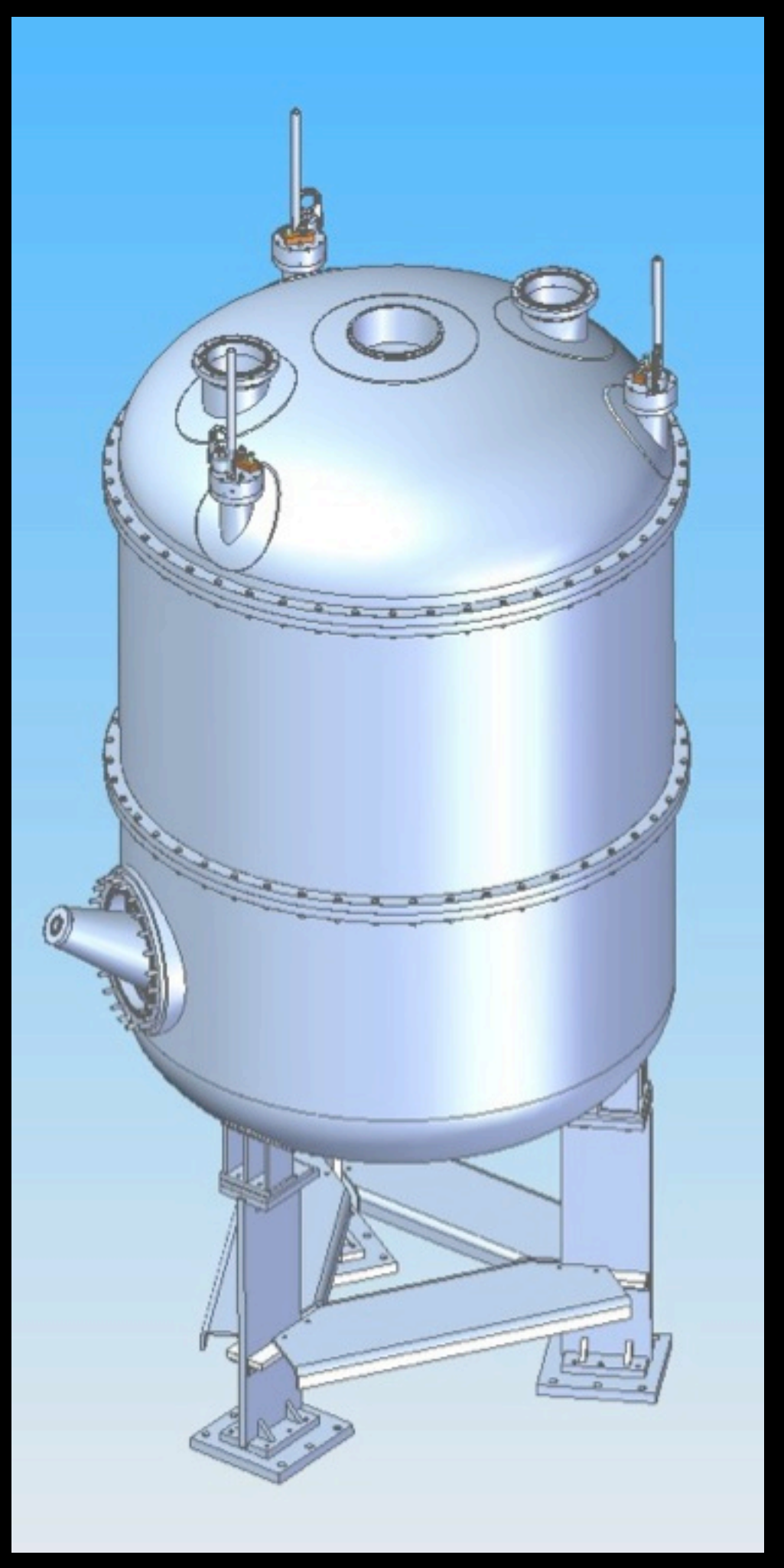




\section{Background suppression by veto}

- Two component outer detector

- Gd-loaded liquid scintillator

- instrumented skin

\section{XeTPC only XeTPC+skin TPC+skin+OD}

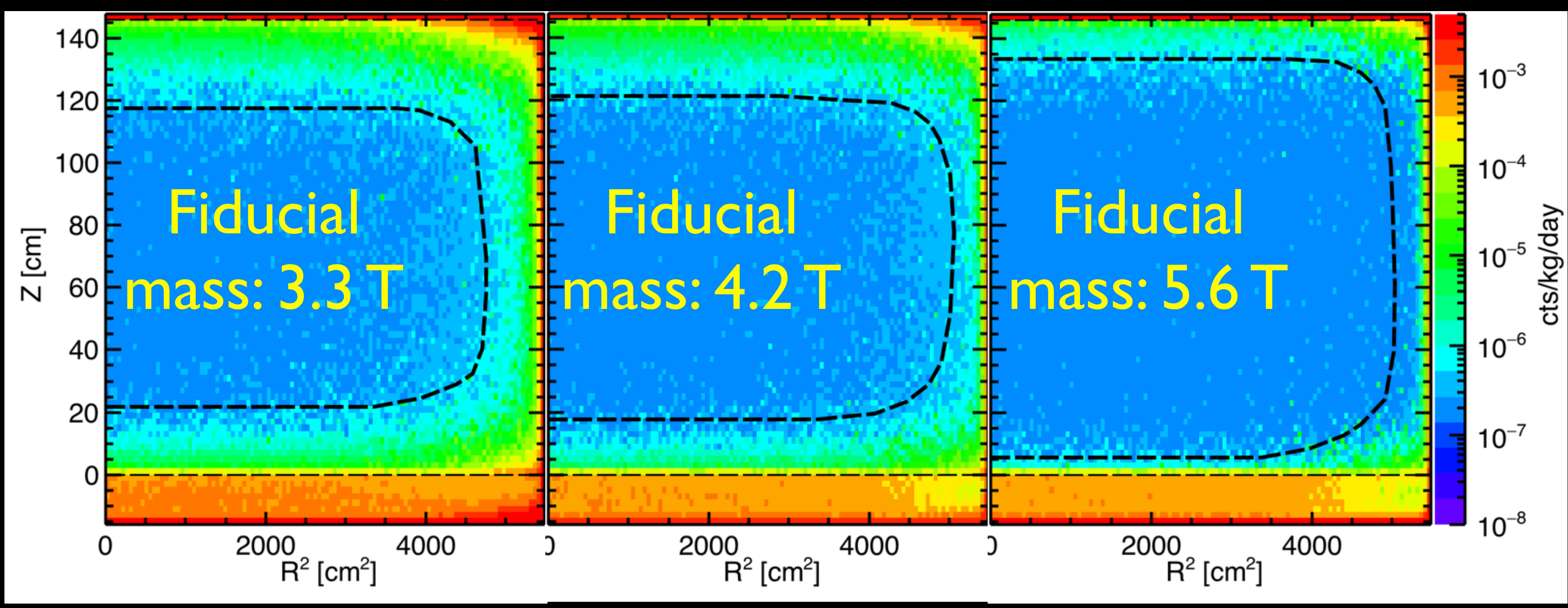




\section{Background suppression by veto}

- Two component outer detector

- GWith veto, detector components are - in a subdominant background!

\section{XeTPC only XeTPC+skin TPC+skin+OD}

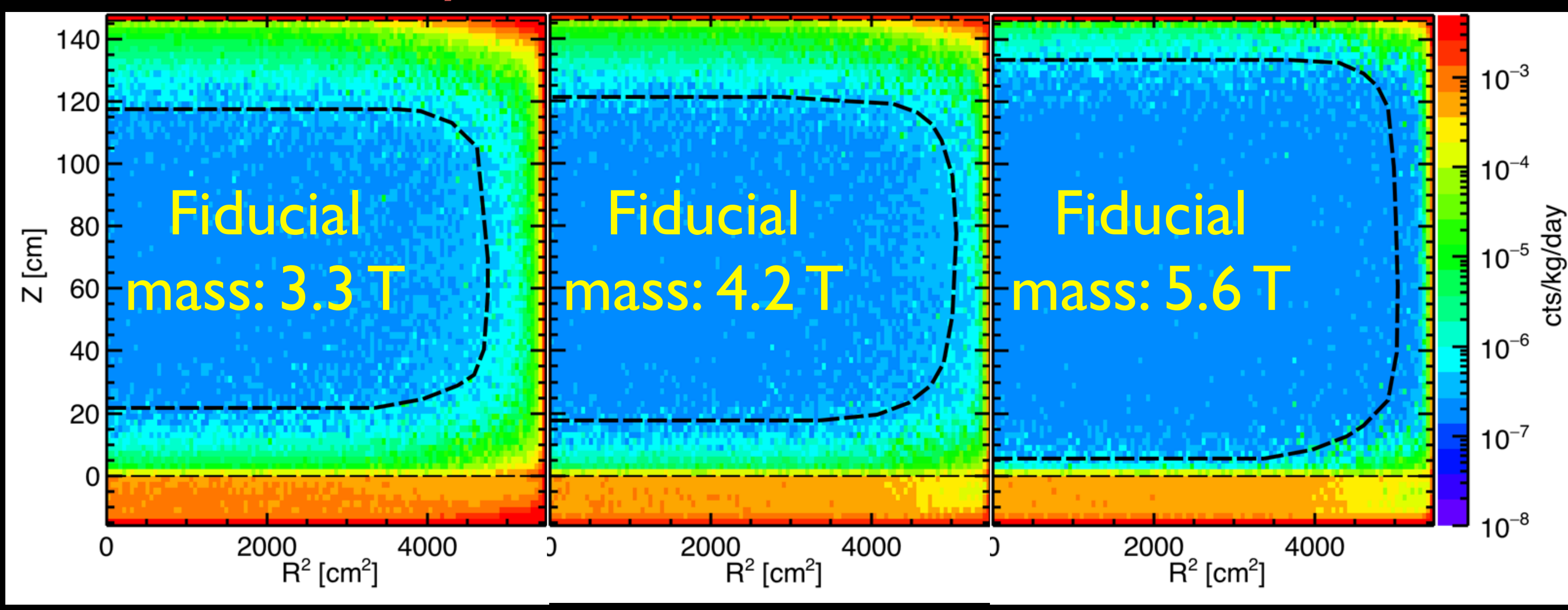


- Radon, Krypton, Argon

\section{Internal backgrounds}

- Distributed throughout the liquid volume

- ER backgrounds (can discriminate, thankfully)

- Radon requirement (goal) of $20(\mathrm{I}) \mathrm{mBq}$

\section{Radon emanation measurements}
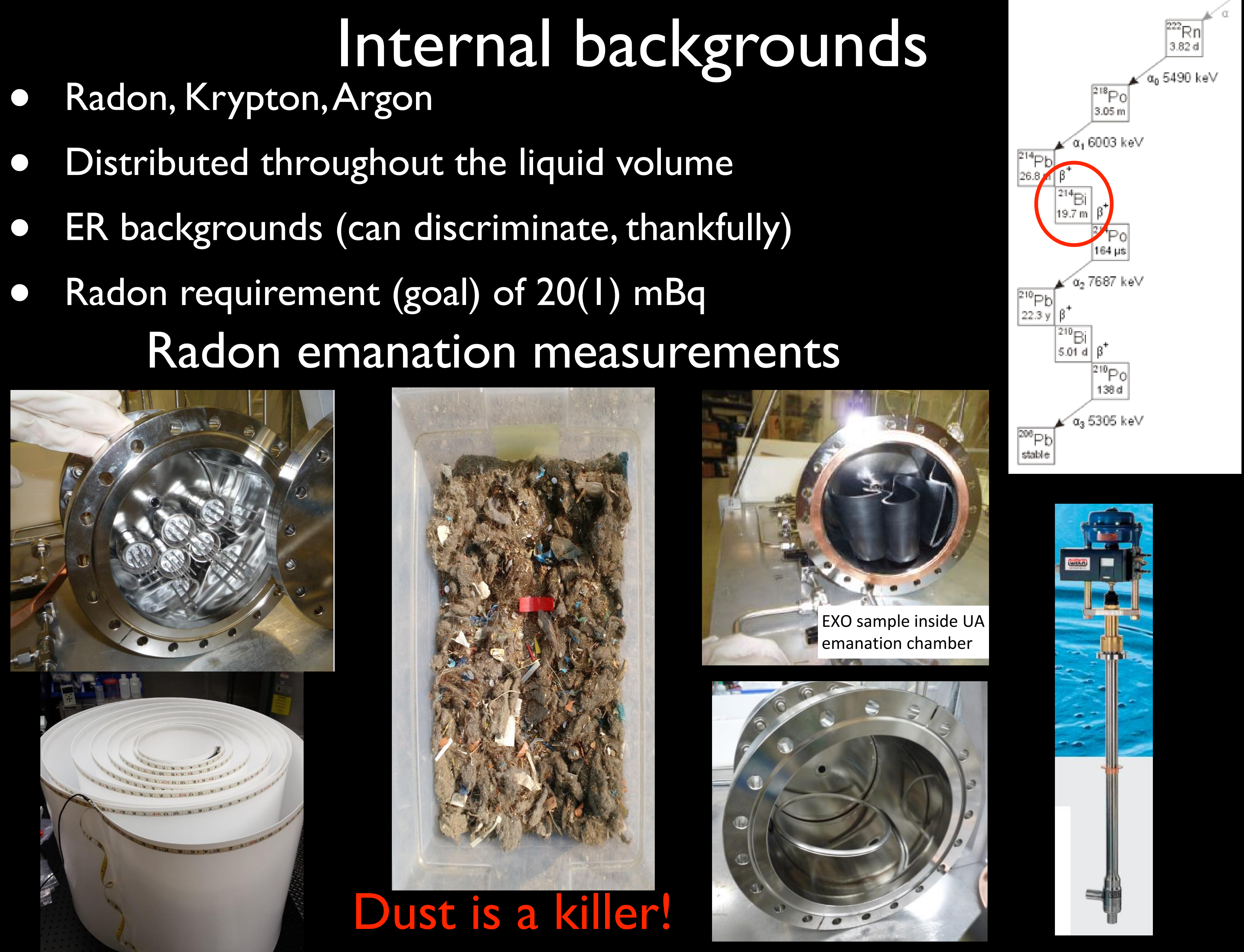

Dust is a killer! 


\section{Internal backgrounds}

- Contributes half our radon budget

- Emanation measurements of "clean room dust"

- Requirement of $<500 \mathrm{ng} / \mathrm{cm}^{2}$ of dust in LZ

- Goal of $5 \mathrm{ng} / \mathrm{cm}^{2}$

- SNO achieved $20 \mathrm{ng} / \mathrm{cm}^{2}$, BOREXINO I $\mathrm{ng} / \mathrm{cm}^{2}$

- I gram total!

- Cleanliness protocols, witness plate protocols, packaging protocols

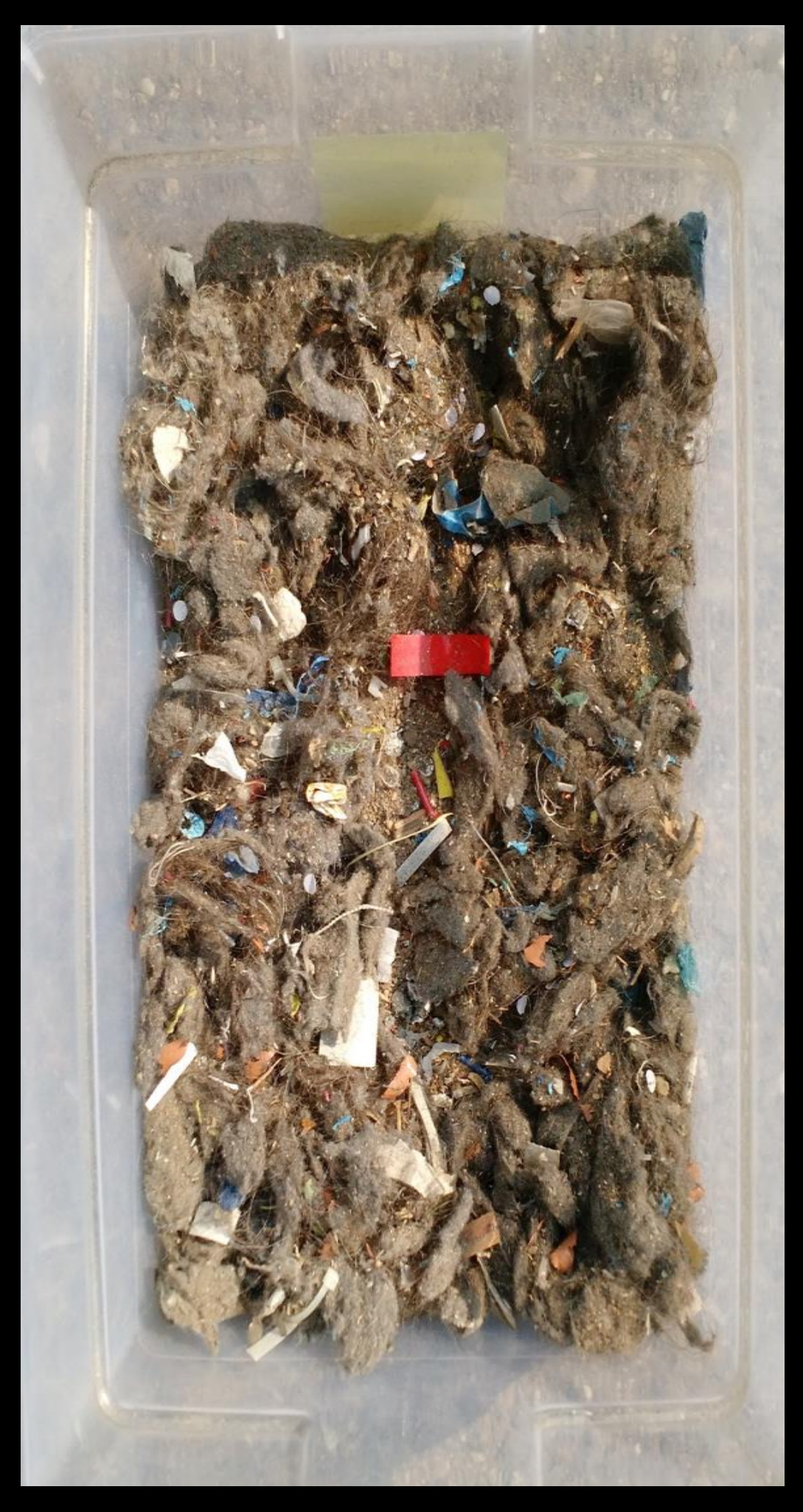

Dust is a killer! 
Intrinsic Contamination Backgrounds Mass (kg) Composite

U early

U late

Th early

Th late

Co60

K40 n/yr (inc.

\begin{tabular}{cc} 
Upper PMT Structure & 40.5 \\
Lower PMT Structure & 69.9 \\
R11410 3" PMTs & 91.9 \\
R11410 PMT Bases * & 2.8 \\
R8778 2" PMTs & 6.1 \\
R8520 Skin 1" PMTs & 2.2 \\
R8520 Skin PMT Bases * & 0.2 \\
PMT Cabling & 103.5 \\
TPC PTFE & 184.0 \\
Grid Wires & 0.75 \\
Grid Holders & 62.2 \\
Field Shaping Rings & 91.6 \\
TPC Sensors & 0.90 \\
TPC Thermometers & 0.06 \\
Xe Recirculation Tubing & 15.1 \\
HV Conduits and Cables & 137.7 \\
HX and PMT Conduits & 199.6 \\
Cryostat Vessel & 2406.1 \\
Cryostat Seals & 33.7 \\
Cryostat Insulation & 23.8 \\
Cryostat Teflon Liner & 26.0 \\
Outer Detector Tanks & 3199.3 \\
Liquid Scintillator & 17640.3 \\
Outer Detector PMTs & 204.7 \\
uter Detector PMT Supports & 770.0 \\
\hline
\end{tabular}

3.90

$\begin{array}{cc}\mathrm{mBq} / \mathrm{kg}) & (\mathrm{mBq} / \mathrm{kg} \\ 0.23 & 0.49\end{array}$

$3.90 \quad 0.23$

2.40

$71.63 \quad 3.20$

$287.74 \quad 75.80$

0.49
0.30

$\begin{array}{ll}75.80 & 28.36\end{array}$

$137.50 \quad 59.38$

16.88

5.19

212.95

108.46

4.75

42.19

29.83

0.02

1.47

0.02

3.31

0.03

0.33

1.20

0.27

$\begin{array}{ll}5.41 & 0.09\end{array}$

$21.09 \quad 13.51$

0.33

0.28

22.89

335.50

90.46

0.18

1.9

38.48

0.23

0.5

2.59

1.25

1.59

0.40

0.11

26.22

$18.91 \quad 18.91$

$0.02 \quad 0.02$

$0.16 \quad 0.39$

$0.01 \quad 0.01$

570

Subtotal (Detector Components)

1.20

0.29

3.22

3.45

0.03

0.02

0.01

395

0.27

$\mathrm{mBq} / \mathrm{k}$
0.38
0.24
2.99
27.93
16.88
4.75
37.62
3.15
0.03
0.49
0.49
0.23
14.15
25.02
0.33
0.6
0.66
0.25
4.24
3.45
0.03
0.06
0.01
388
0.49

$\mathrm{mBq} / \mathrm{kg}$

MBolkg

S.F. rej.j)

ER (cts) $\mid N R$

\begin{tabular}{lll|l}
1.46 & 2.53 & 0.05 & 0.000
\end{tabular}

\begin{tabular}{lll|l|l}
16.25 & 412.50 & 52.80 & 0.13 & 0.008
\end{tabular}

\begin{tabular}{lll|l|l}
24.20 & 332.76 & 4.60 & 0.02 & 0.001
\end{tabular}

\begin{tabular}{lll|l|l}
2.23 & 123.61 & 3.62 & 0.00 & 0.000
\end{tabular}

\begin{tabular}{lll|l|l}
0.65 & 33.14 & 2.65 & 1.43 & 0.000
\end{tabular}

\begin{tabular}{lll|l|l}
0.00 & 0.12 & 22.54 & 0.06 & 0.008
\end{tabular}

$\begin{array}{lll}1.60 & 0.40 & 0.02\end{array}$

$\begin{array}{lll}1.60 & 0.40 & 6.33\end{array}$

$\begin{array}{lll}0.00 & 0.54 & 10.83\end{array}$

$0.50-26.29 \quad 24.77$

$7.26 \quad 3,359$

$\begin{array}{lll}7.05 & 0.30\end{array}$

1.4

1.24

0.07

10.03

1.97

0.00

0.04

0.00

0.00

222Rn $(2.0 \mu \mathrm{Bq} / \mathrm{kg})$

220Rn $(0.1 \mu \mathrm{Bq} / \mathrm{kg})$

natKr $(0.015 \mathrm{ppt} \mathrm{g/g})$

natAr (0.45 ppb g/g)

210Bi $(0.1 \mu \mathrm{Bq} / \mathrm{kg})$

Laboratory and Cosmogenics

Fixed Surface Contamination

\section{Subtotal (Non-v counts)}

Physics Backgrounds

136Xe $2 v \beta \beta$

Astrophysical $v$ counts $(p p+7 B e+13 N)$

Astrophysical $v$ counts (8B)

Astrophysical $v$ counts (Hep)

Astrophysical v counts (diffuse supernova)

Astrophysical v counts (atmospheric)

Subtotal (Physics backgrounds)

Total

Total (with 99.5\% ER discrimination, 50\% NR efficiency)

My summary of the summary table $\longrightarrow$

6 ER, $0.6 \mathrm{NR}$ in 1000 days! 


\section{Backgrounds summary}

\begin{tabular}{l}
\hline Subtotal (Non-v counts) \\
\multicolumn{1}{|c|}{ Physics Backgrounds } \\
\hline 136 Xe $2 \mathrm{v} \beta \beta$ \\
Astrophysical v counts (pp+7Be+13N) \\
Astrophysical v counts (8B) \\
Astrophysical v counts (Hep) \\
Astrophysical v counts (diffuse supernova) \\
Astrophysical v counts (atmospheric) \\
\hline Subtotal (Physics backgrounds) \\
\hline Total \\
\hline Total (with $99.5 \%$ ER discrimination, 50\% NR efficiency) \\
\hline
\end{tabular}

\begin{tabular}{|c|c|}
\hline $\mathbf{9 2 1}$ & $\mathbf{0 . 5 0}$ \\
\hline \multicolumn{2}{|c|}{} \\
\hline 67 & 0 \\
255 & 0 \\
0 & $0^{* *}$ \\
0 & 0.21 \\
0 & 0.05 \\
0 & 0.46 \\
\hline 322 & 0.72 \\
\hline 1,240 & 1.22 \\
\hline 6.22 & 0.61 \\
\hline
\end{tabular}

- Lots of neutrinos - significant fraction of both ER and NR counts

- Discrimination cuts are important 


\section{Sensitivity projections}

\begin{tabular}{|l|c|c|c|}
\hline Detector Parameter & Reduced & Baseline & Goal \\
\hline Light collection (PDE) & 0.05 & 0.075 & 0.12 \\
\hline Drift field $(\mathrm{V} / \mathrm{cm})$ & 160 & 310 & 650 \\
\hline Electron lifetime $(\mu \mathrm{s})$ & 850 & 850 & 2800 \\
\hline PMT phe detection & 0.8 & 0.9 & 1.0 \\
\hline $\mathrm{N}-$ fold trigger coincidence & 4 & 3 & 2 \\
\hline${ }^{222} \mathrm{Rn}(\mathrm{mBq}$ in active region) & 13.4 & 13.4 & 0.67 \\
\hline Live days & 1000 & 1000 & 1000 \\
\hline
\end{tabular}

- $\sim 6 \mathrm{keVnr}$ threshold in baseline scenario (LUX achieved $4.5 \mathrm{keVnr}$ )

- Driven by SI trigger coincidence threshold

- Better than $99.5 \%$ ER/NR discrimination at this field 


\section{Sensitivity projections (1000 days)}

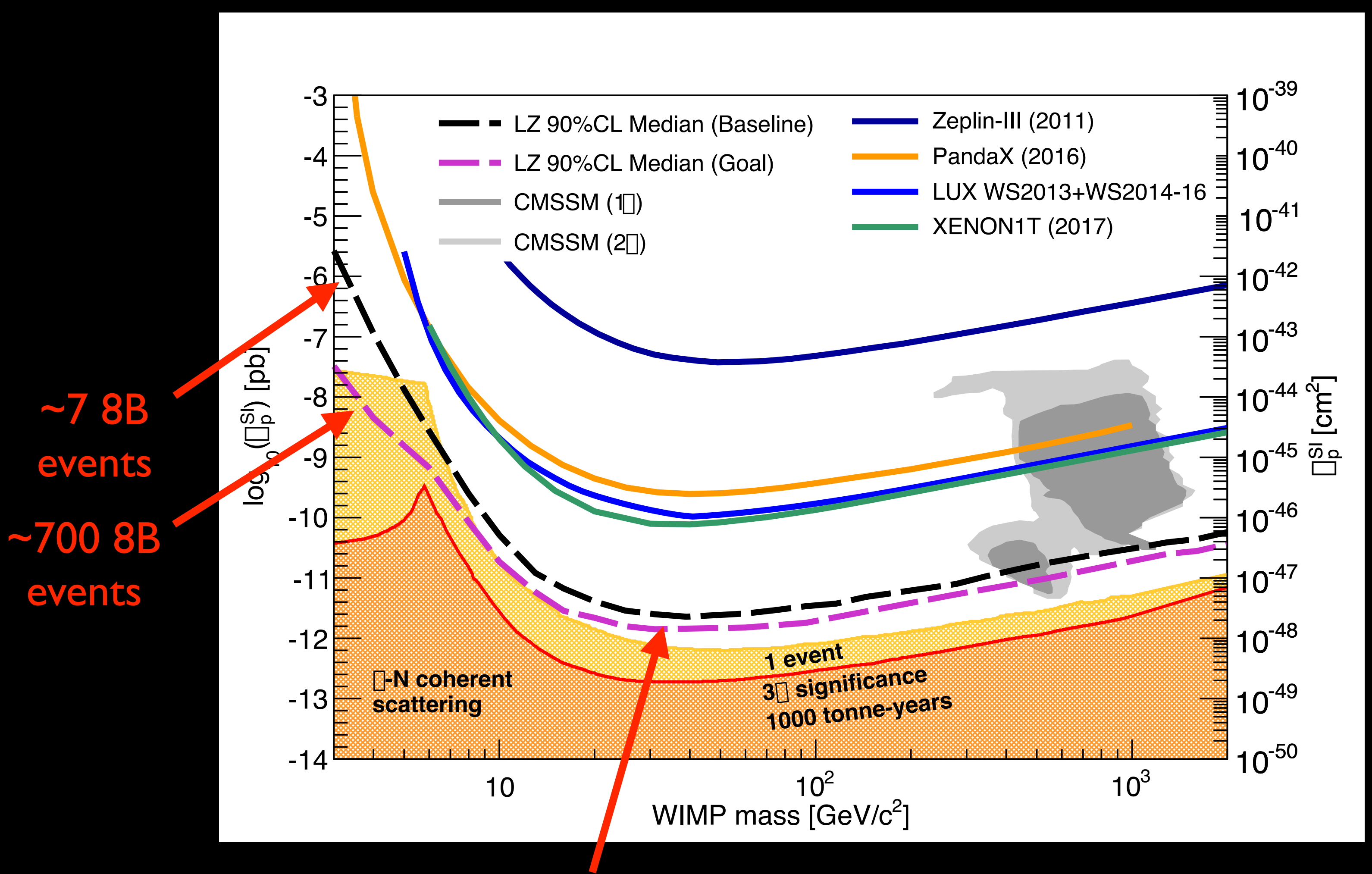

@40 GeV: 2.3e-48 Nominal

I. I e-48 Goal 


\section{WIMP signal region}

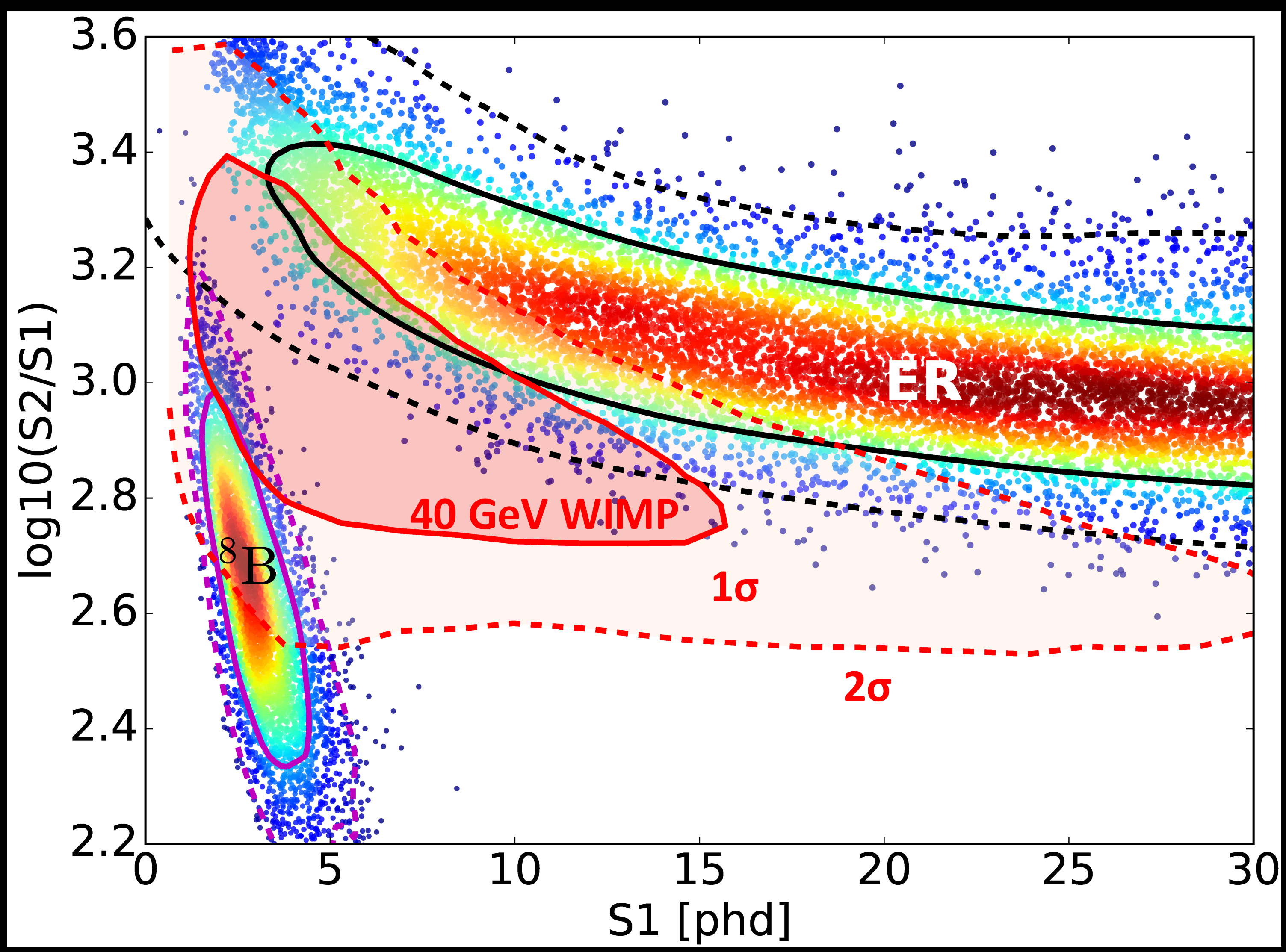




\section{Sensitivity projections (1000 days)}

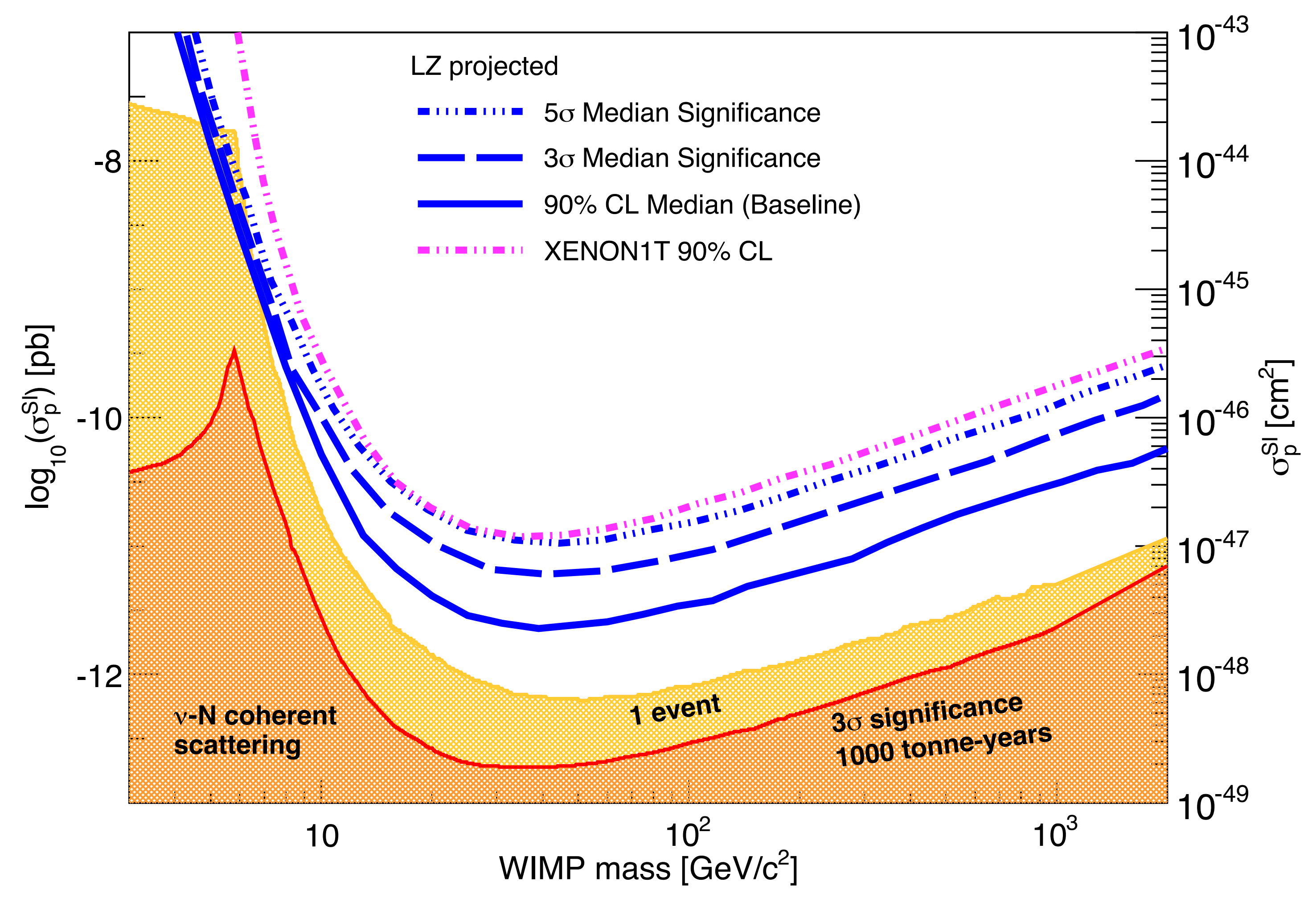




\section{Schedule}

- 2012 - LZ Collaboration formed

- 2014 - LZ Project start

- 2015 - DOE CD-I approval - Conceptual Design Report (1509.02910)

- 2016 - DOE CD-3 approval - Technical Design Report (I703.09/44)

- March 2017 - LUX removed from water tank

- 2018 - Underground construction begins

- 2019 - Commissioning 


\section{Schedule}

- Competition is fierce!

- XENONIT out with new results, already heading to XENONnT

- Infrastructure already in place - update of TPC and cryostat

- PandaX also has a strong group

- We're moving as fast as we can! 


\section{Summary}

- Liquid xenon TPCs are the leading technology in the search for $\sim 10 \mathrm{GeV}$ and above WIMPs (spin independent)

- Mature technology, challenge is to make the detectors bigger

- Scaling up raises new technical questions (HV, internal radioactivity, ...)

- $\mathrm{LZ}$ is poised to achieve a factor $>30$ more sensitivity than current best limits

- The race is on for the next order of magnitude in sensitivity 
End 


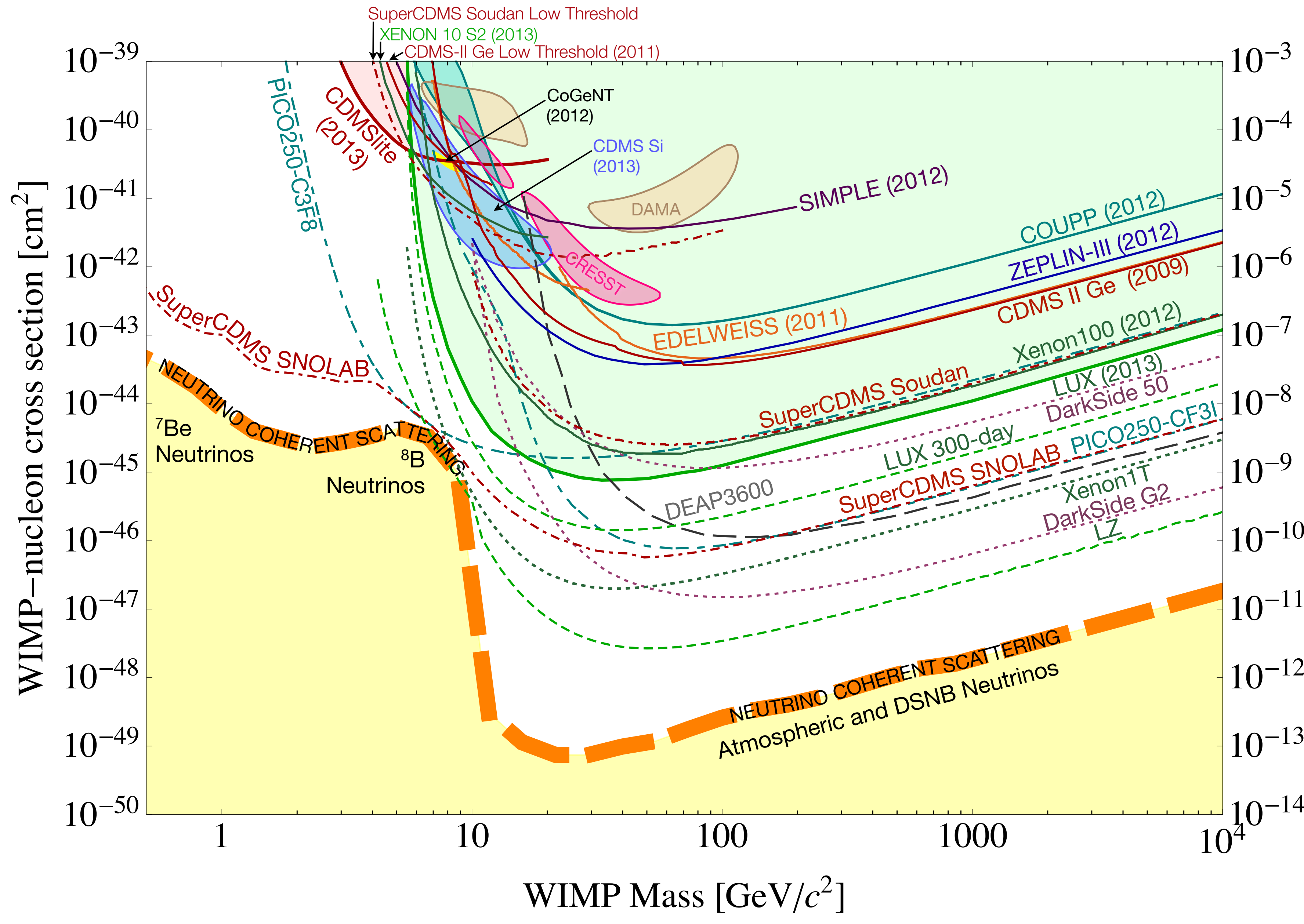


Dark Matter Searches: Past, Present \& Future

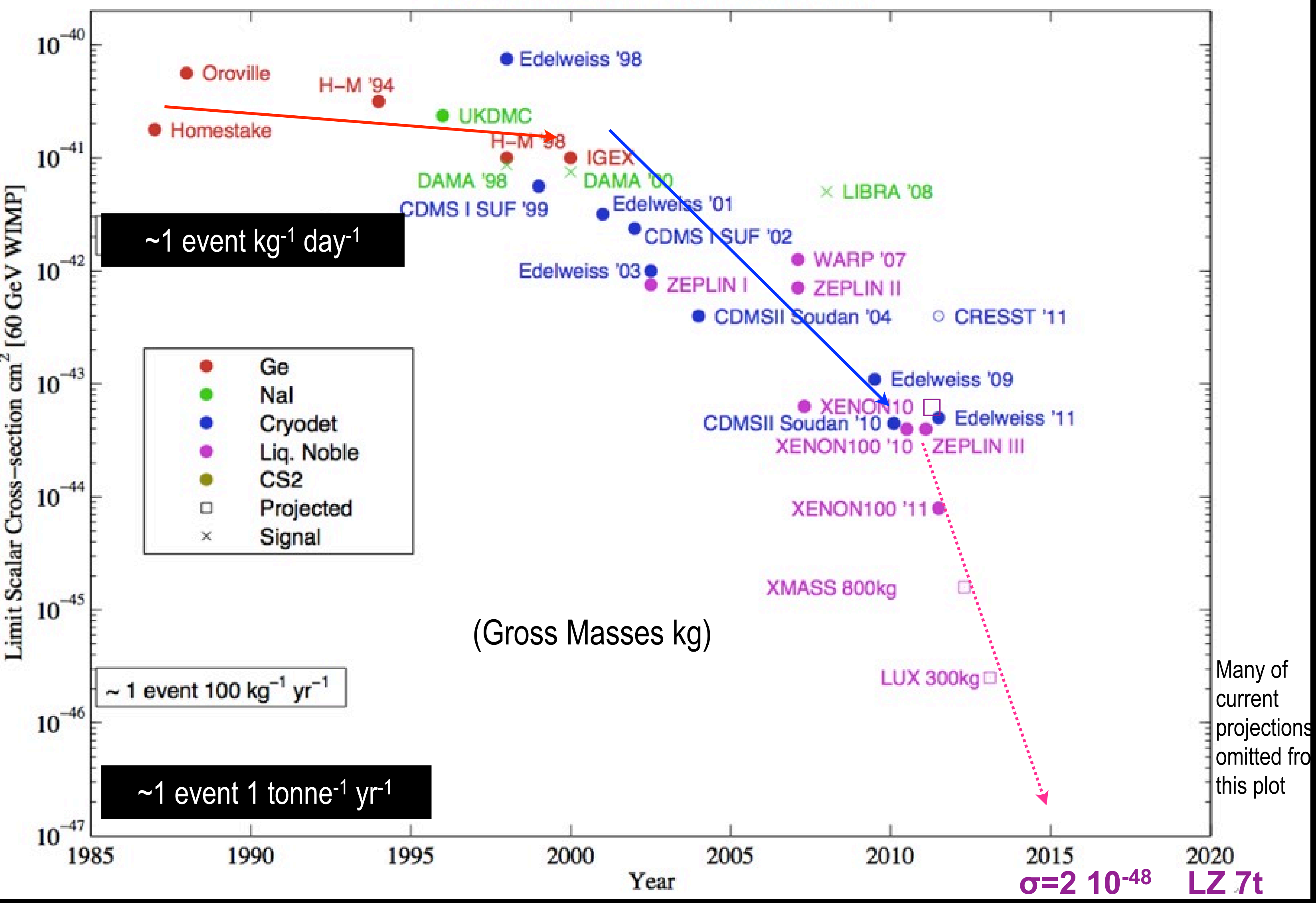




\section{Some LXe physics}

\section{Electron/Nuclear}

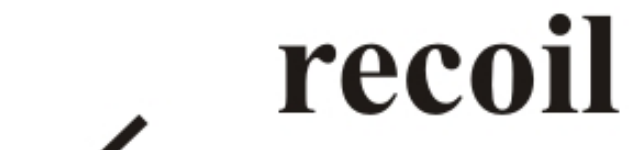

Excitation
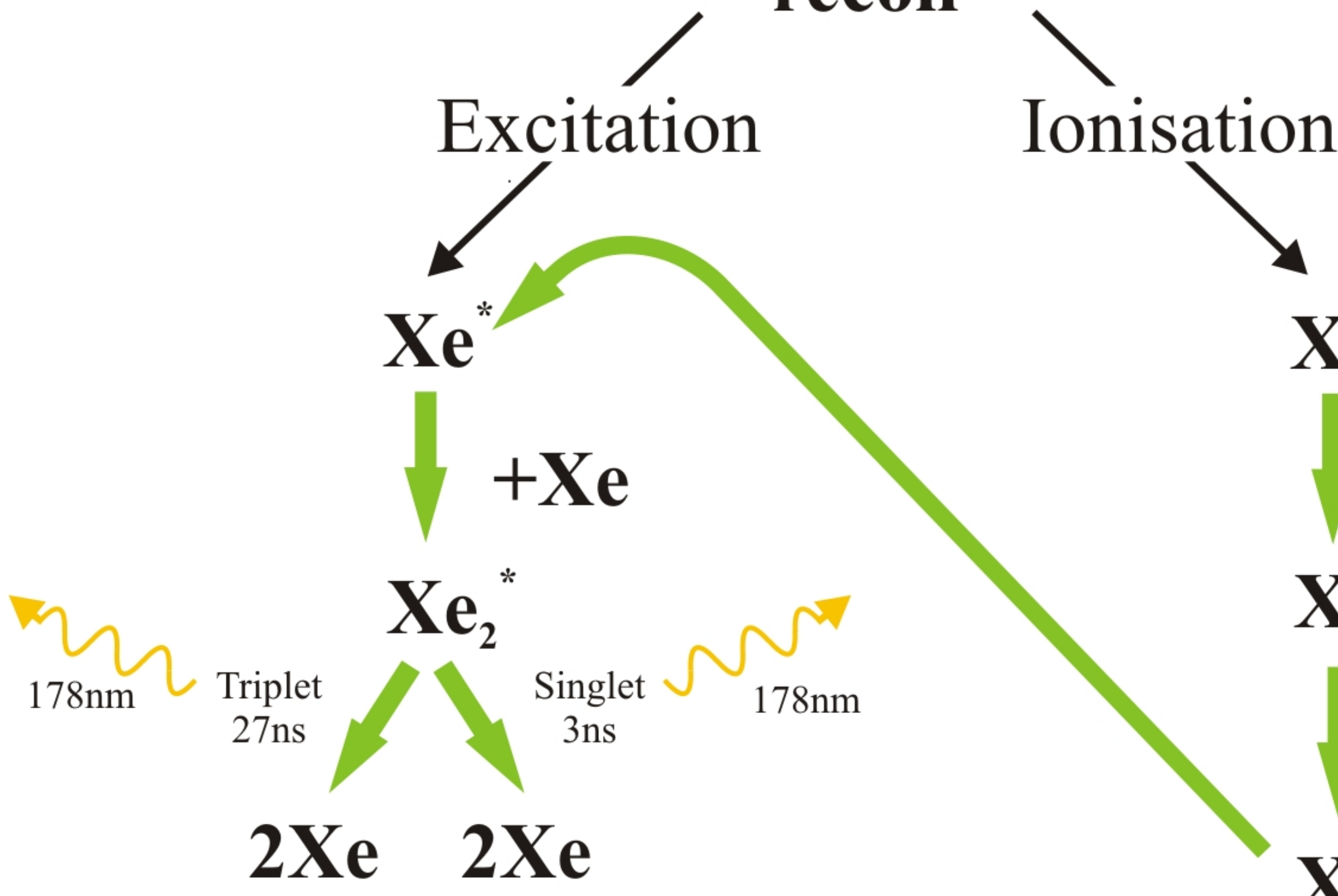

$\mathrm{Xe}^{+}$

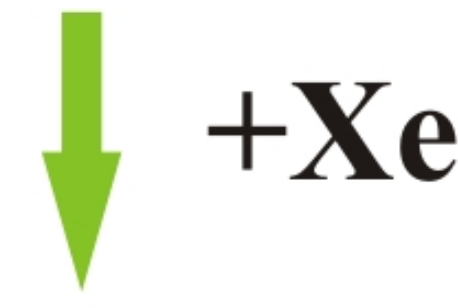

$\mathrm{Xe}_{2}^{+}$

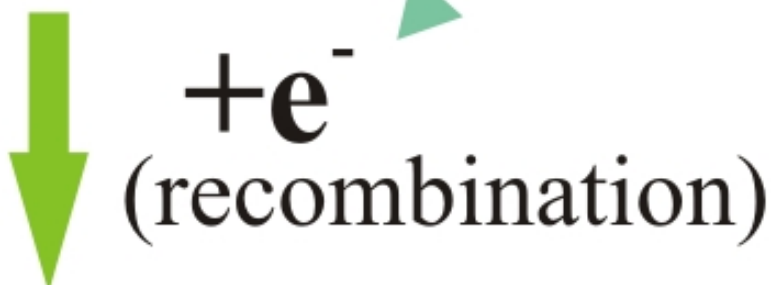

$\mathrm{Xe}^{* * *}+\mathrm{Xe}$ 


\section{Some LXe physics}

- Significant difference between ER and NR tracks

- ER lead to more signal than NR

- More NR energy goes into heat and is lost

- Lindhard factor, Leff, Quenching factor

- Two energy scales $k e V_{\text {ee }}$ and $\mathrm{keV}_{\mathrm{nr}}$

- Leads to different behavior with field

- Also leads to ER/NR discrimination

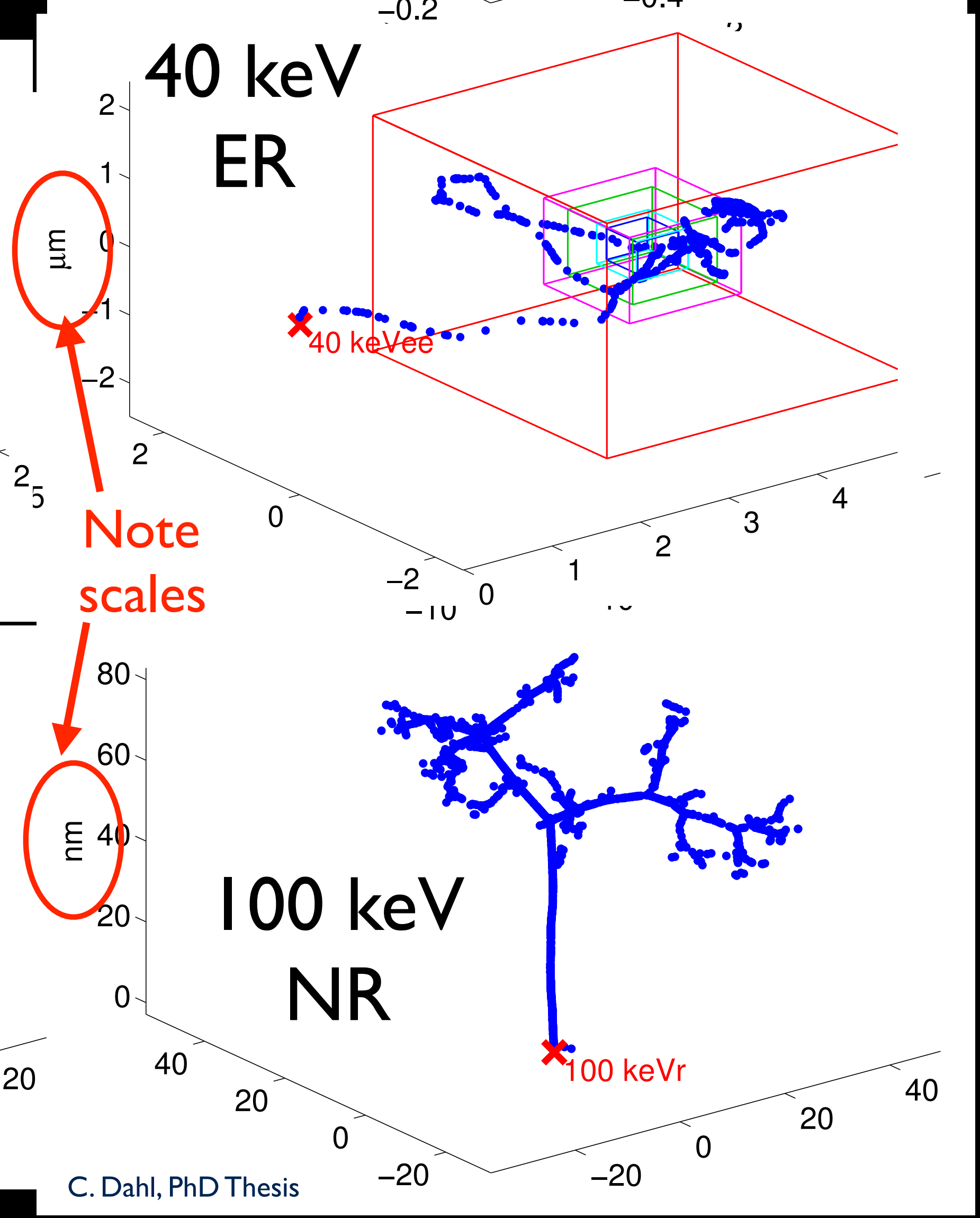




\section{Requires calibration}

- LUX has really done great work here

- Kr-83m - Over le6 events spread uniformly throughout detector

Fiducial volume determination

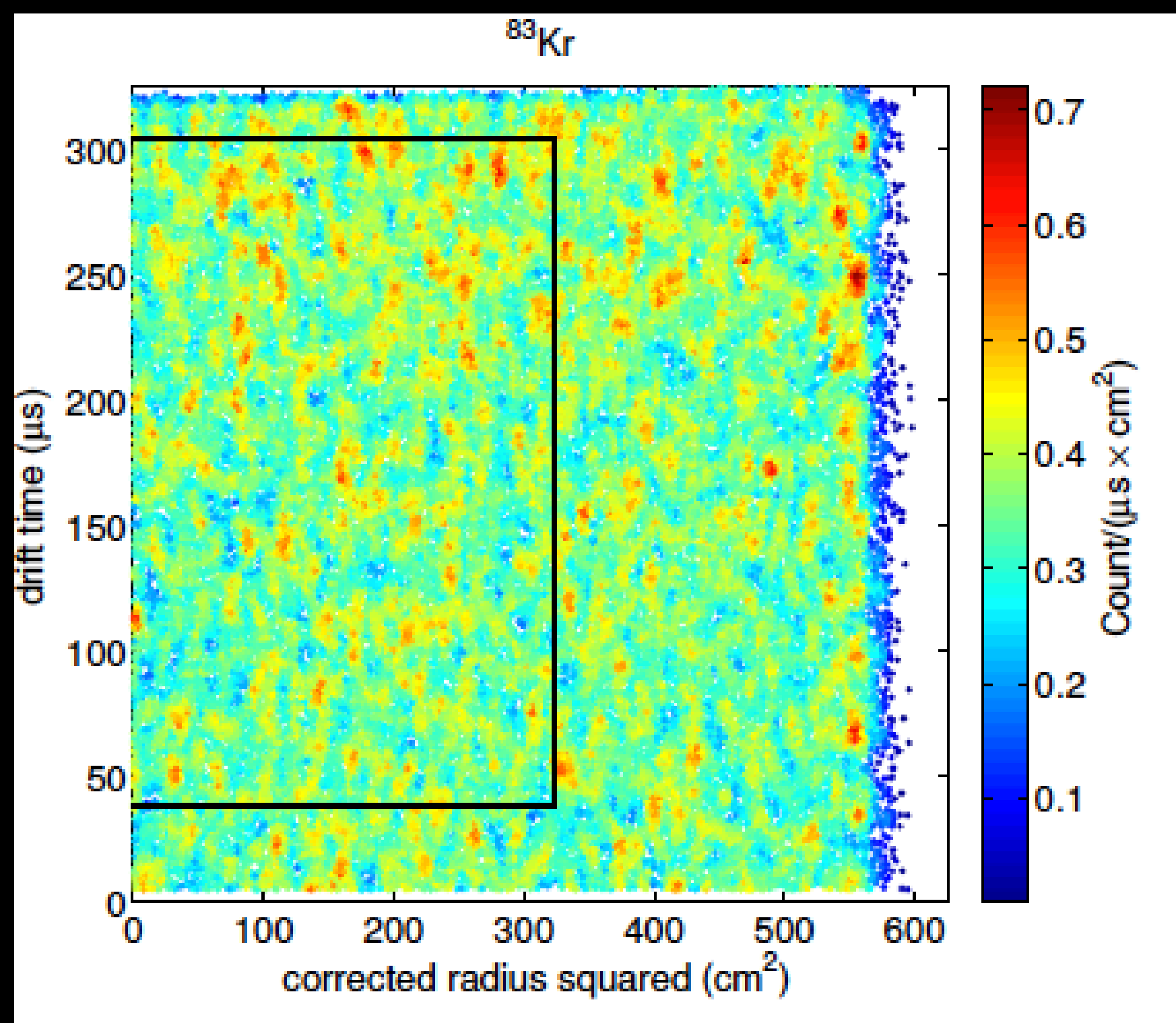

\section{Position-based SI corrections}



\section{Requires calibration}

- LUX has really done great work here

- DD neutron generator to measure NR yields
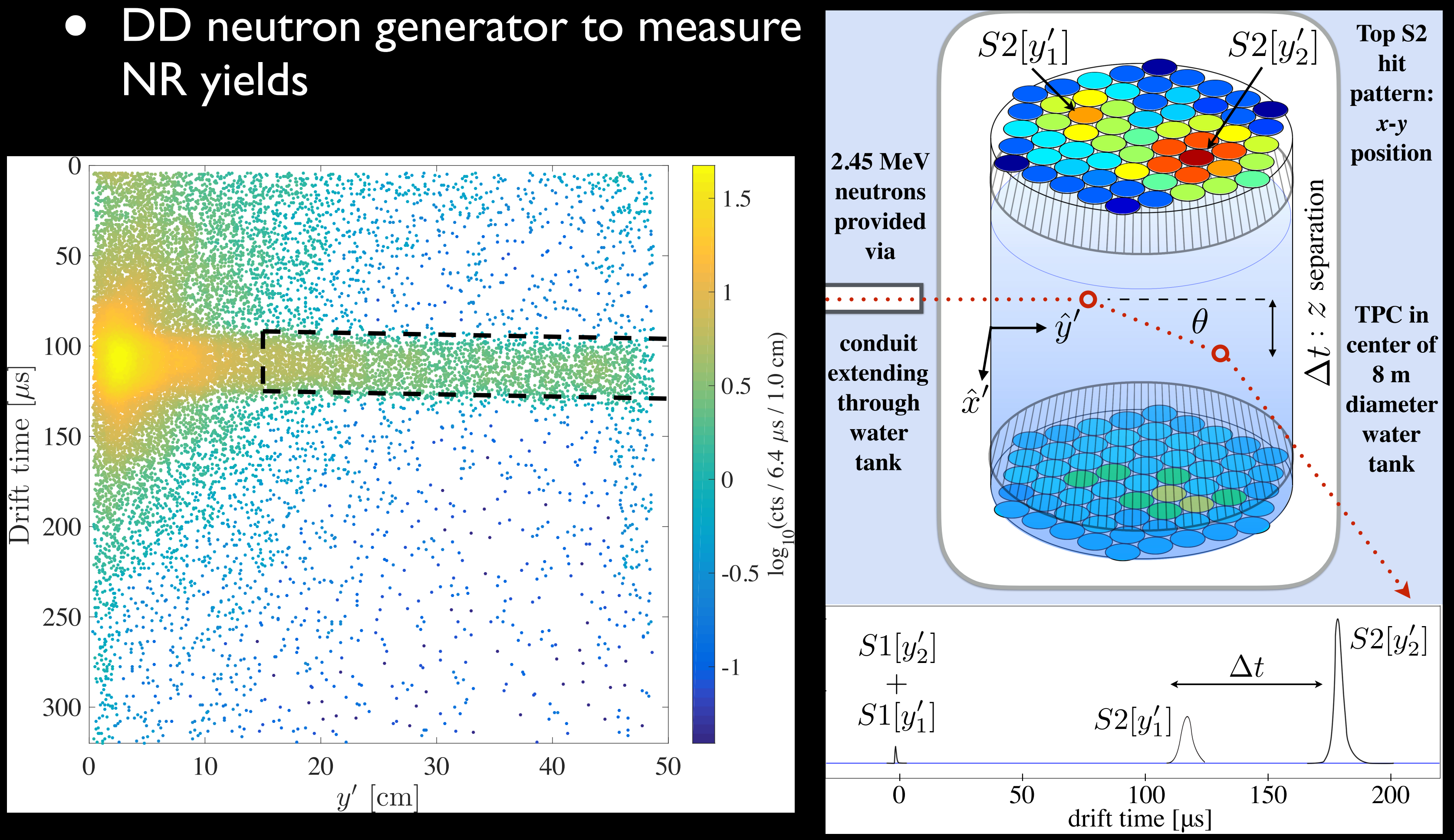


\section{Requires calibration}

- LUX has really done great work here

- DD neutron generator to measure NR yields

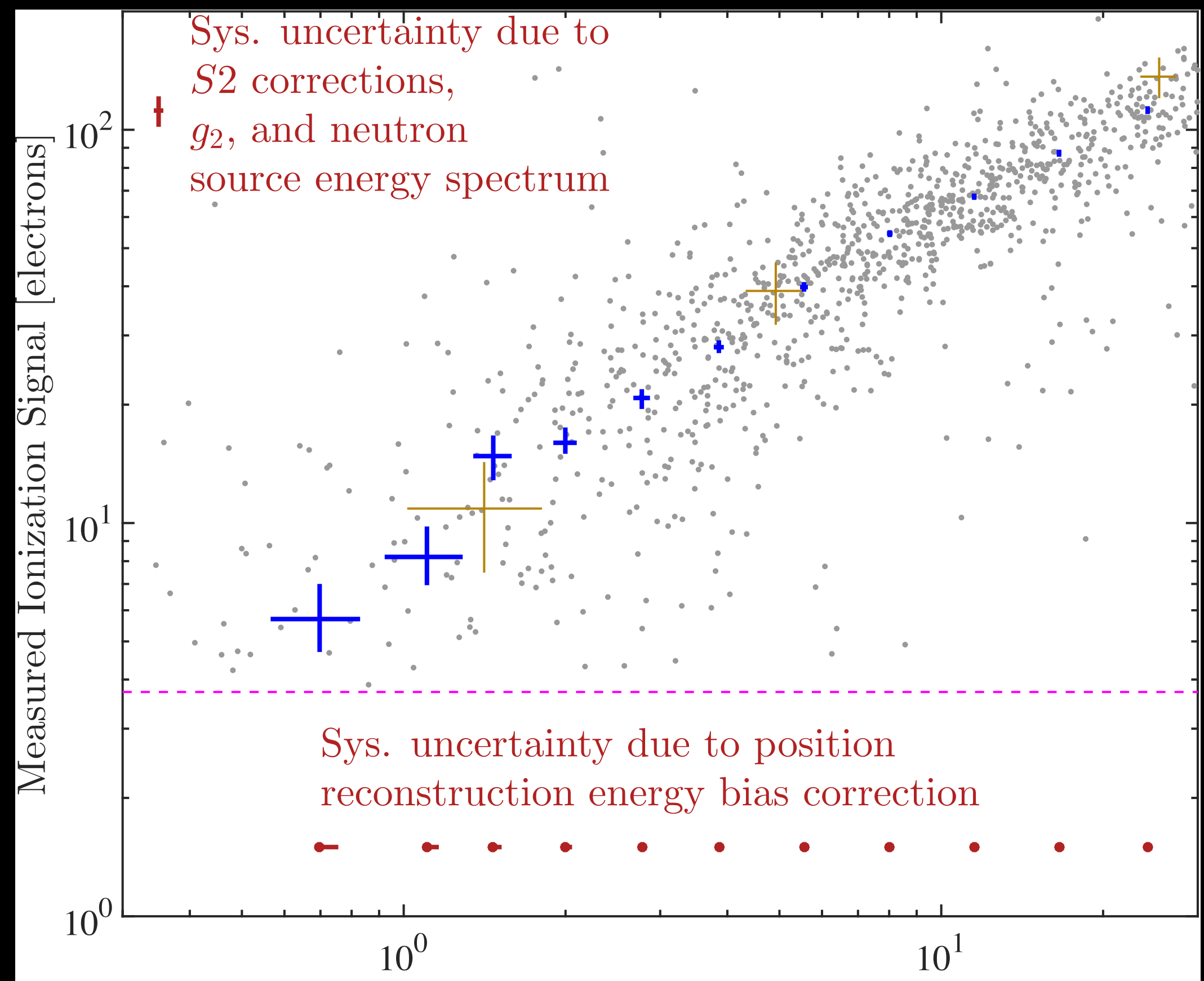

Nuclear Recoil Energy [keVnr] 


\section{Leads to background rejection}

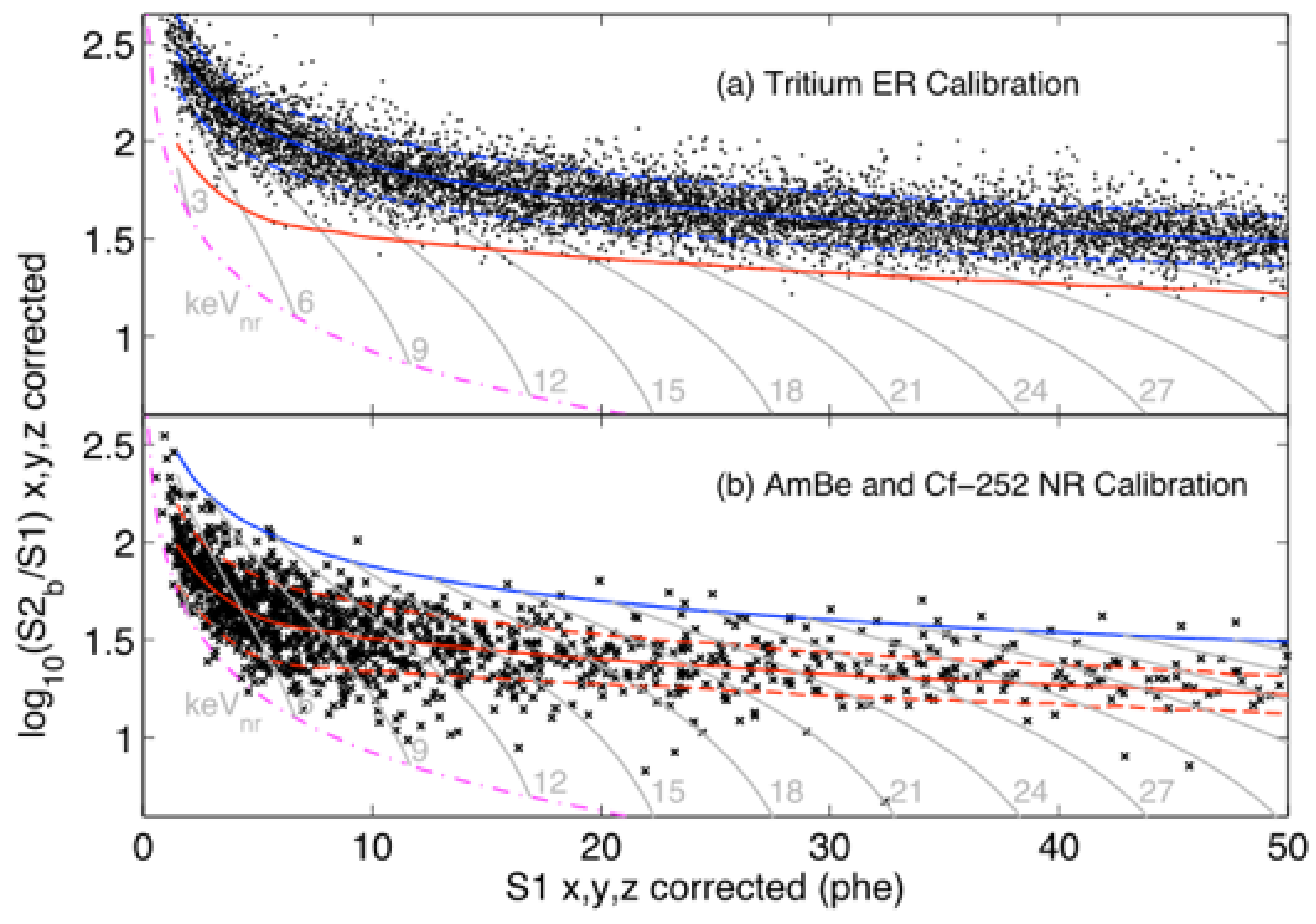

Grey contours indicate lines of constant energy 


\section{Some LXe physics}

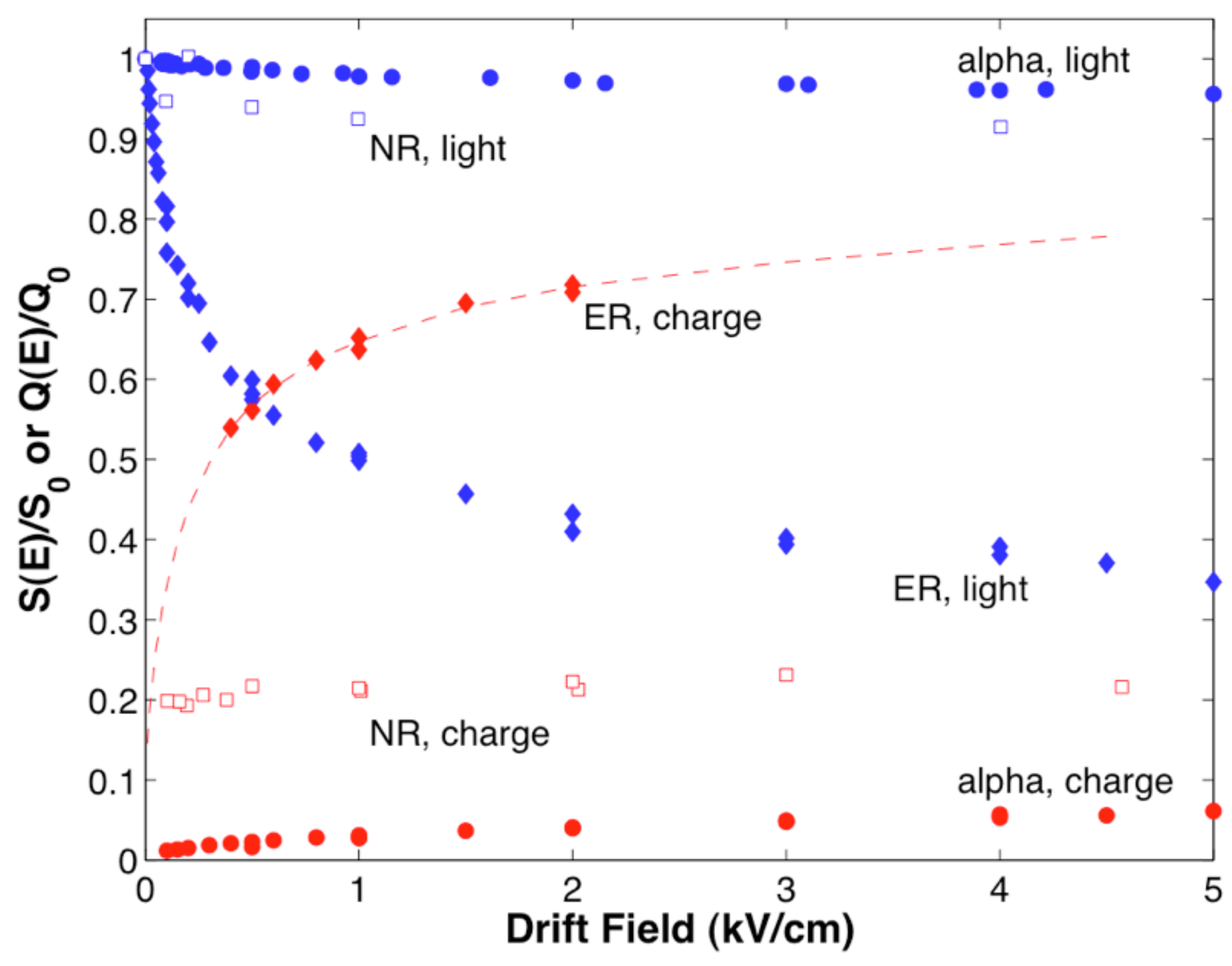

E. Aprile et al., Phys. Rev. Lett. 97, 081302 (2006)

For I 22 keV ER, 56 keV NR 


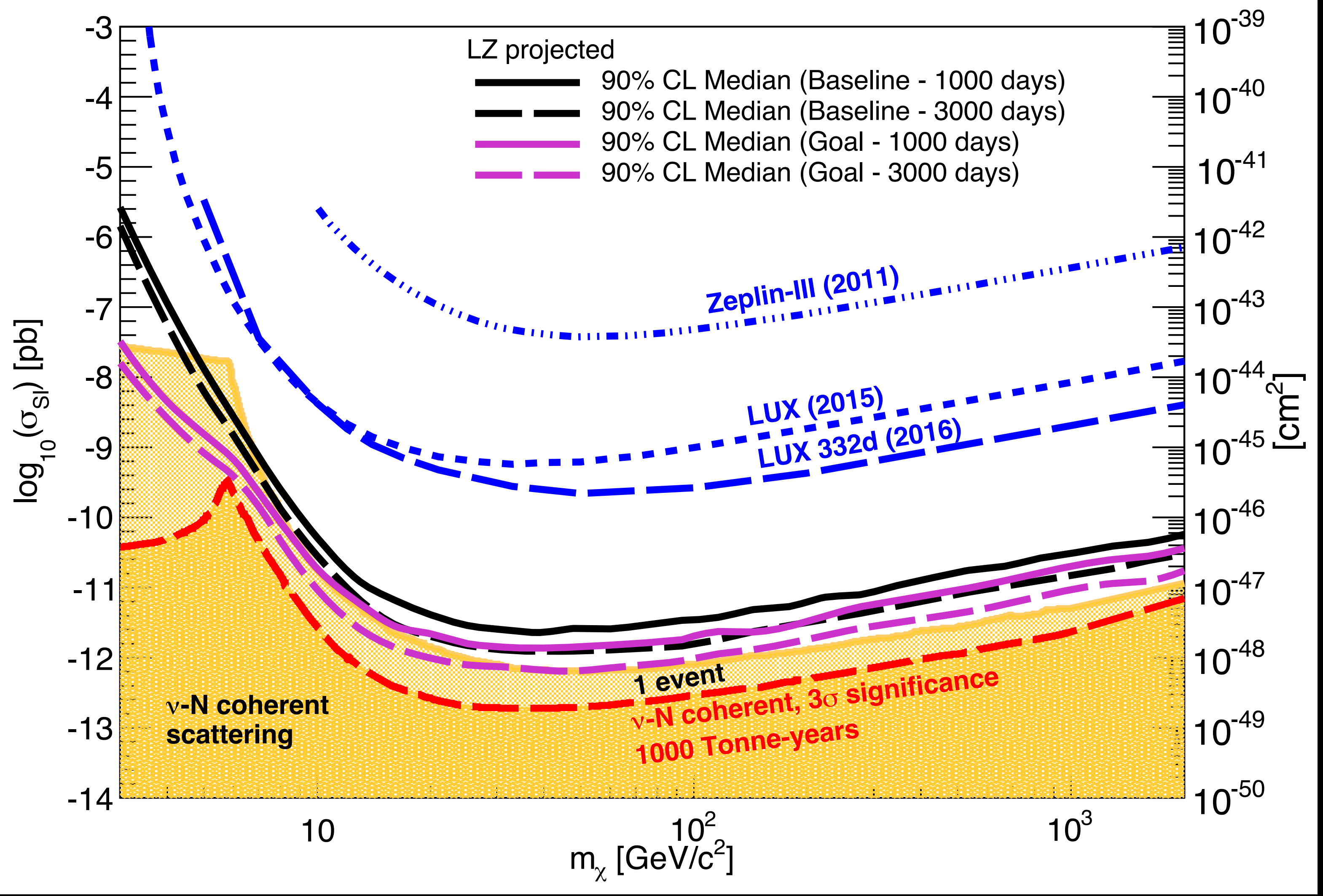




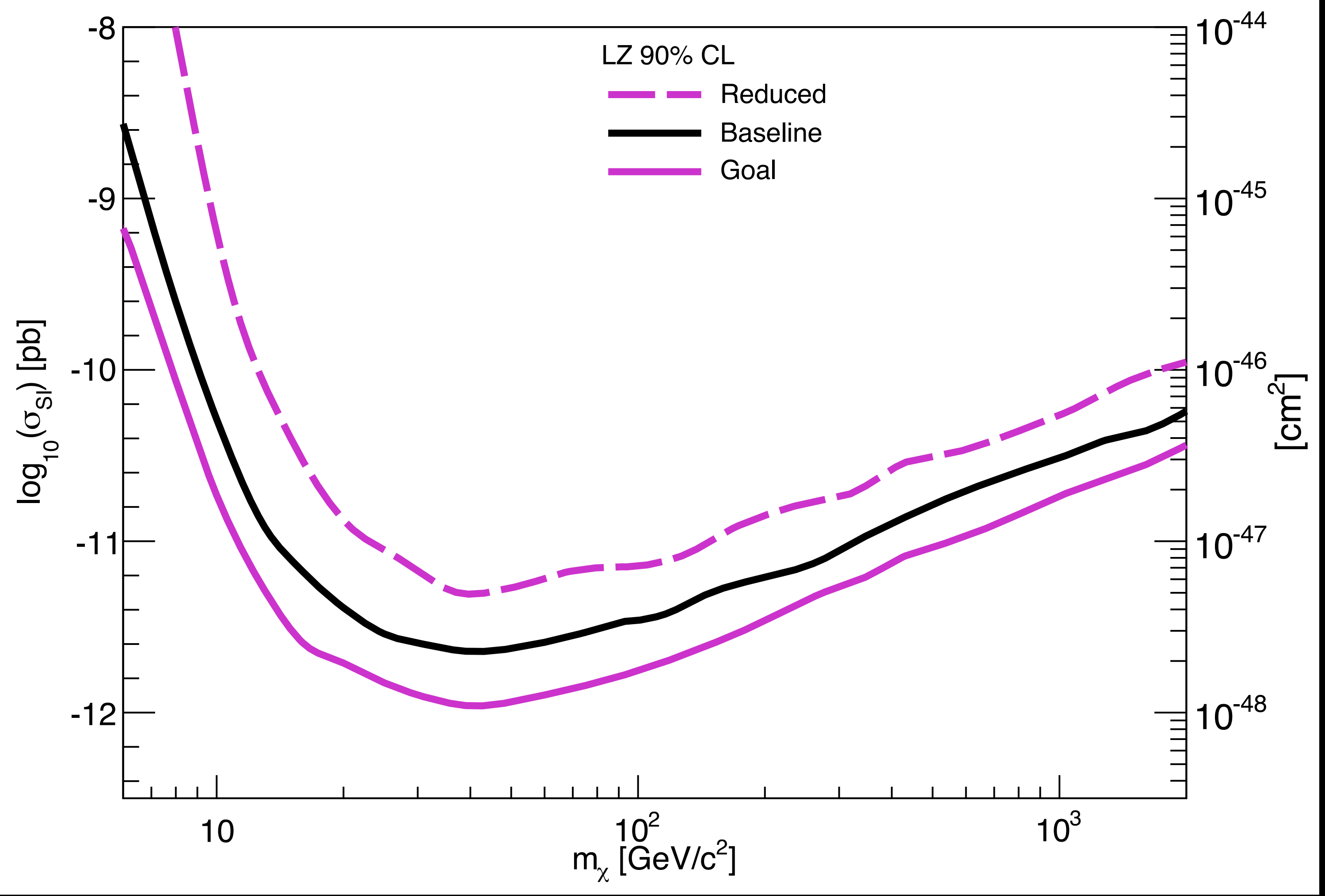




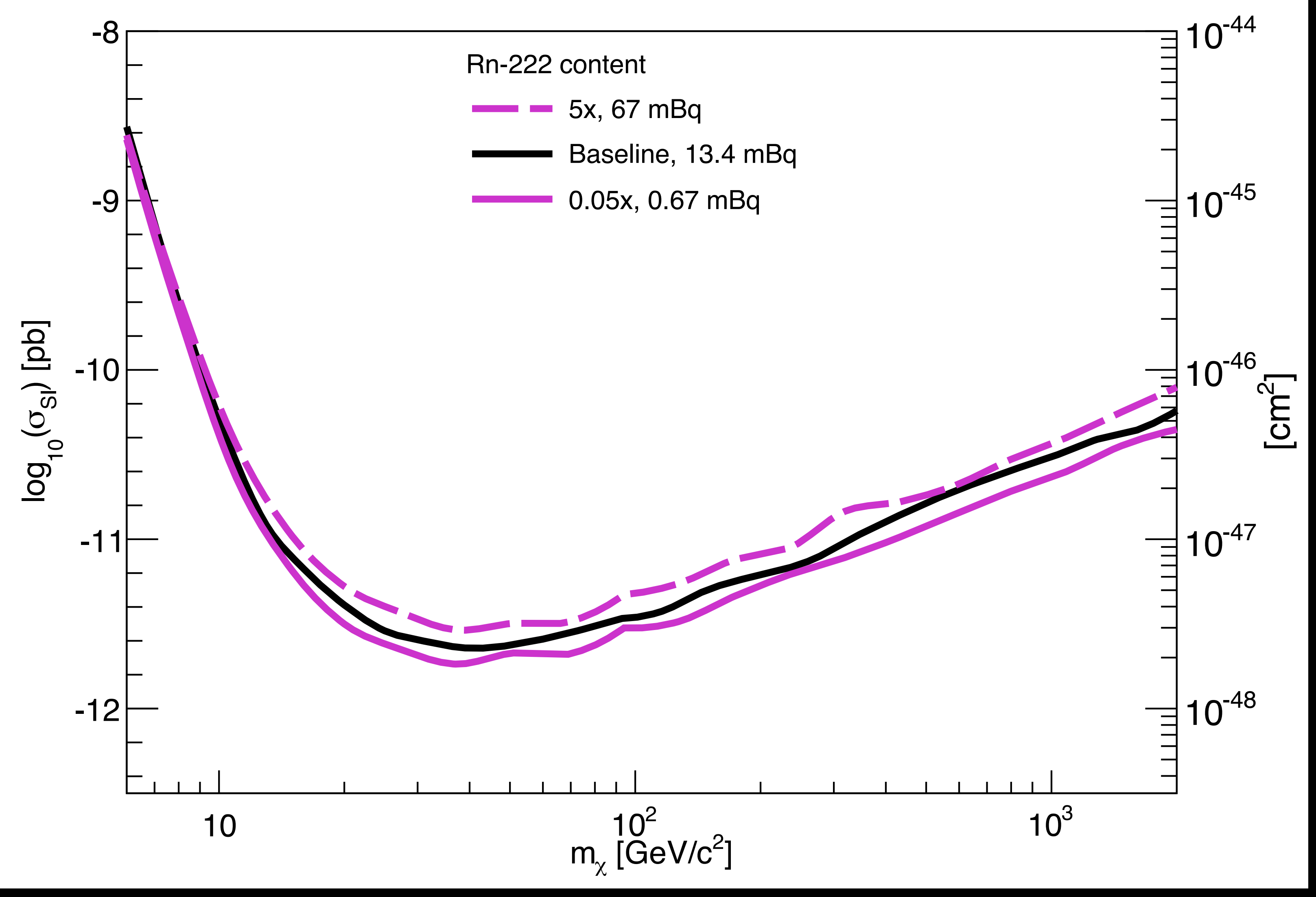




\section{PLR (Profile Likelihood Ratio)}

- Simple fiducial of $5600 \mathrm{~kg}$ (X,Y,Z position info not yet implemented in PLR)

- Dominant ER: Rn, Kr, pp-neutrinos spatially uniform like signal
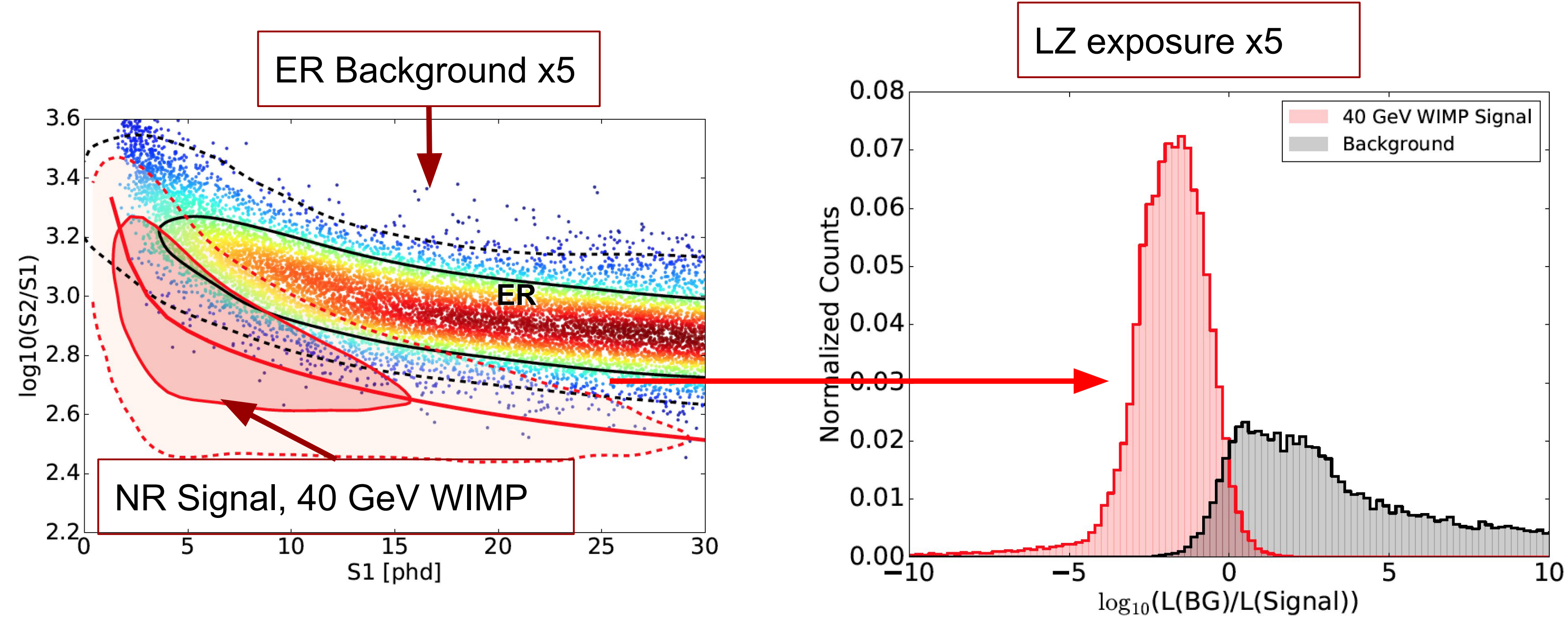